\title{
The local stellar population of nova regions in the Large Magellanic Cloud
}

\author{
A. Subramaniam and G. C. Anupama \\ Indian Institute of Astrophysics, II Block Koramangala, Bangalore 560 034, India \\ Received 29 January 2002 / Accepted 13 May 2002

\begin{abstract}
This study aims at identifying and understanding the parent population of novae in the Large Magellanic Cloud (LMC) by studying the local, projected, stellar population. The star formation history of the local environment around novae is studied based on photometric data of stars and star clusters in the nova neighbourhood, available in the OGLE II survey and star cluster catalogues. The age of the stellar population within a few arcmin around nova regions are estimated using isochrone fits to the $V$ vs. $(V-I)$ colour-magnitude diagrams. The fraction of stars in various evolutionary states are compared using luminosity functions of the main-sequence stars and the red giant stars.

The age, density and luminosity function of the stellar population are estimated around 15 novae. The upper limit of the age of the intermediate stellar population is found to be $4 \mathrm{Gyr}$ in all the regions, excepting the region around the slow nova LMC 1948. Star formation in these regions is found to have started between 4-2.0 Gyr ago, with a majority of the regions starting star formation at 3.2 Gyr. This star formation event lasted upto 1.6-0.8 Gyr.

The star formation history of the underlying population of both the fast and moderately fast novae indicate their parent population to be similar and likely to be in the age range 3.2-1.0 Gyr. This is in good agreement with the theoretical age estimates for Galactic cataclysmic variables.

The region around the slow nova shows a stellar population in the age range 1-10 Gyr, with a good fraction older than 4 Gyr. This indicates that the progenitor might belong to an older population, consistent with the idea that the progenitors of slow novae belong to older population.
\end{abstract}

Key words. stars: novae, cataclysmic variables - galaxies: Magellanic Clouds - galaxies: stellar content

\section{Introduction}

Novae belong to the class of cataclysmic variables (CVs), i.e. interacting close binary systems in which a white dwarf accretes matter from a Roche lobe filling companion. Novae undergo outbursts of amplitude $\Delta m=8-15$ in the optical range, reaching magnitudes as high as $M_{v} \sim-9$. The outbursts, which are accompanied by ejection of matter with velocities $\geq 300 \mathrm{~km} \mathrm{~s}^{-1}$, are triggered by a thermonuclear runaway reaction in the hydrogen burning shell at the bottom of the accreted layer on the surface of the white dwarf. The rate of decline of the nova, which is well correlated with the outburst maximum, determines the speed class of the nova. The brightness of novae together with the well established relation between the absolute magnitude at maximum and the rate of decline make these objects valuable as secondary standards for extragalactic distance measurements (e.g. Capacciolli et al. 1989; Capaccioli et al. 1990; Pritchet \& van den Bergh 1987; Della Valle \& Livio 1995). The use of novae as distance indicators is

Send offprint requests to: A. Subramaniam, e-mail: purni@iiap.ernet.in however complicated by the lack of understanding of how the properties of novae vary between galaxies.

The photometric and spectroscopic development of a nova outburst depend on the properties of the accreting white dwarf such as its mass and composition (CO or ONeMg type) and also on other factors such as the metallicity in the accreted material and the accretion rate (see Kato 1997; Starrfield et al. 2000). Some or all these factors may depend on the underlying stellar population. A study of the underlying population in a nova environment would hence be useful in revealing the influence of the various parameters on nova properties.

Attempts to study the progenitors of novae have, in the past, been based on their spatial distribution (eg. Duerbeck 1984; Ciardullo et al. 1987; Della Valle \& Duerbeck 1993; Della Valle et al. 1994). Population synthesis models of the statistics and properties of Galactic CVs and extragalactic novae indicate that the rate of formation of CVs, the nova rate and the distribution of novae over speed classes depend on the star formation history (SFH) (Yungelson et al. 1997 and references therein). Based on an analysis of the speed classes of Galactic and extragalactic novae and their spatial distribution, Duerbeck (1990) and Della Valle et al. (1992) suggested the presence of two 
nova populations: fast, bright disk novae and slow, faint bulge novae. They also suggested the disk novae originate from more massive white dwarfs. Hatano et al. (1997) and Hatano et al. (1997) investigated the observability and spatial distribution of classical novae in the Galaxy and M31 using a Monte Carlo technique together with a simple model for the distribution of dust, and found that most novae in these galaxies come from the disk population rather than the bulge population. Further, the bulge-to-disk nova ratio is similar to the overall bulge-todisk mass ratio of the galaxy. Galaxies such as M33 and the Large Magellanic Cloud (LMC) are disk dominated galaxies. Hence most of the nova population in these galaxies are expected from the disk population.

A study of the underlying stellar population can be made by understanding the SFH of the region around novae using clusters and field stars in the region. Such a study of the environmental effects on the nova population in the Galaxy is influenced by the fact that it is not very easy to identify their parent population and the age due to (a) the nova distribution is observed from within the Galaxy, and (b) mixing of different stellar population in the disk of the Galaxy. To study the environmental effects on novae and the implications, it is hence ideal to look at an external galaxy where the uncertainty in internal distances are eliminated. The LMC is one of the very few external galaxies where a large number of novae have been detected and studied in detail over the years. The known differences in the abundances and evolution between the LMC and the Galaxy might help in understanding the influence of these parameters on novae properties. Further, the LMC is believed to have undergone a burst of star formation 3-5 Gyr ago, which probably continued to the present day (Butcher 1977). This event of star formation resulted in the majority of the intermediate population seen in the disk of the LMC. Thus, a study of the SFH may be able to point to the age of the parent population of novae in the LMC.

Nova outbursts in the LMC have been recorded since 1926 (Buscombe \& de Vaucouleurs 1955). A study of the distribution of novae and supernova remnants in the Magellanic Clouds by van den Bergh (1988) indicated novae were widely distributed over the face of the LMC with a possible clumping slightly to the south of the Bar. van den Bergh also noted that there was no concentration of novae within the Bar itself, in contrast with the distribution of supernova remnants, and concluded the Bar to be younger than the intermediate age population with which novae are associated. Subsequent to van den Bergh's study, some of the novae detected in the recent past are found to be located in the region of the Bar.

In the present work, the stellar population in the region of novae are studied using star clusters and field stars in the nova neighbourhood. Star clusters are used to trace the epochs of cluster formation while field stars trace the SFH of the region. This study, which is the first such attempt, thus aims at identifying and understanding the properties of the parent population of novae by studying the local SFH of the novae regions instead of the global SFH of the LMC. Such a study will enable inter-comparison of the properties such as the age and metallicity of the different regions, which may be used to compare and correlate with the properties of novae.

\section{Novae in the LMC and their distribution}

Thirty novae have so far been discovered in the LMC, of which one is a recurrent nova. Only 19 novae have photometric/spectroscopic data from which the speed class and other outburst properties are available. Following the speed classes given by Duerbeck (1990), we find that 14 novae belong to the very fast class $\left(t_{3}<10\right)$, or fast $\left(t_{3}=11-25\right.$ days $)$ category. Among these, one is super bright (LMC 1991), one is recurrent (LMC 1968/LMC 1990\#2) and three are known to be ONeMg novae (LMC 1981, LMC 1988\#2, LMC 1990\#1). 3 novae belong to the moderately fast category $\left(t_{3}=26-80\right.$ days $)$ and two have been found to be slow novae ( $t_{3}>80$ days). LMC 1995 was discovered as a super soft x-ray source at late phases and is analysed to have occurred on a massive $\left(1.2 M_{\odot}\right) \mathrm{CO}$ white dwarf (Orio \& Greiner 1999). The recently discovered LMC 2002 appears to be a fast nova. LMC 1999 does not appear to be a nova. The location, speed class and other known properties of the LMC novae are tabulated in Table 1.

Since the previous study of the distribution of novae in the LMC by van den Bergh (1988), ten new novae and one recurrent nova have been discovered. Figure 1 shows the location of all novae in the LMC, identified by their serial number in Table 1. From the figure, it is seen that novae are widely distributed over the face of the LMC, with a lack of novae in the 30 Dor region, and an apparent clumping of novae towards the south east of the bar.

\section{Novae neighbourhood stellar population}

The ages, density and metallicity of the projected stellar population in the neighbourhood of novae are studied based on the star clusters and the field star population in that region. As the focus of this study is the intermediate age population, the recent ground based surveys of the LMC can be used to obtain the data on stellar population. Various, extensive surveys have generated a large database of star clusters and field stars in the LMC. For the present analyses, the data on field stars are obtained from the OGLE II survey (Udalski et al. 2000). The data from only one survey are used since it is essential to have a homogeneity of data for an inter-comparison of the different regions. All the available catalogues of the LMC star clusters are used to locate clusters in the neighbourhood of novae.

\subsection{Data and analyses}

\subsubsection{Field stars}

Field stars within a radius of a few arcmin in the vicinity of novae were identified from the OGLE II survey. Photometric data were found to be available for regions around 15 novae. As more observations were found to be available in the $I$ passband, the $V$ vs. $(V-I)$ colour-magnitude diagrams (CMDs) were used in the analyses. All CMDs show a fairly narrow main-sequence (MS) and a tight red giant branch (RGB) and a red giant clump (RGC). The structure of the RGB and the RGC are looked at in greater detail in order to analyse the properties of the intermediate age stellar population in the region, to which the novae presumably belong. 
Table 1. List of novae in the Large Magellanic Cloud: coordinates, speed class and type, references for speed class and type.

\begin{tabular}{|c|c|c|c|c|c|c|c|c|c|c|}
\hline \multirow[t]{2}{*}{ No. } & \multirow[t]{2}{*}{ Nova } & \multicolumn{3}{|c|}{ RA (2000) } & \multicolumn{3}{|c|}{ Dec (2000) } & \multirow{2}{*}{$\begin{array}{l}t_{3} \\
\text { Days }\end{array}$} & \multirow[t]{2}{*}{ Remarks } & \multirow[t]{2}{*}{ Ref. $^{a}$} \\
\hline & & $\mathrm{h}$ & $\mathrm{m}$ & $\mathrm{s}$ & $\circ$ & , & $"$ & & & \\
\hline 1. & LMC 1926 & 05 & 14 & 54.54 & -66 & 48 & 44.06 & 200 & slow nova & $1,2,3$ \\
\hline 2. & LMC 1935 & 03 & 59 & 15.90 & -67 & 46 & 35.71 & 25.1 & fast nova & $1,2,3$ \\
\hline 3. & LMC 1936 & 05 & 07 & 26.75 & -66 & 39 & 12.08 & 31.6 & moderately fast nova & $1,2,3$ \\
\hline 4. & LMC 1937 & 05 & 57 & 04.44 & -68 & 54 & 47.92 & 19.9 & fast nova & $1,2,3$ \\
\hline 5. & LMC 1948 & 05 & 38 & 15.38 & -70 & 20 & 26.23 & 101.1 & slow nova & $1,2,3$ \\
\hline 6. & LMC 1951 & 05 & 12 & 51.93 & -69 & 58 & 36.25 & 6.26 & v. fast nova & $1,2,3$ \\
\hline 7. & LMC 1968 & 05 & 09 & 58.28 & -71 & 39 & 51.49 & 5.26 & v. fast nova; USco type RN & $2,3,4,5$ \\
\hline 8. & LMC 1970\#1 & 05 & 33 & 13.25 & -70 & 35 & 04.41 & & poor data & 2 \\
\hline 9. & LMC 1970\#2 & 05 & 35 & 28.90 & -70 & 47 & 14.32 & 15.3 & fast nova & 2,3 \\
\hline 10. & LMC 1971\#1 & 04 & 58 & 23.23 & -68 & 05 & 34.02 & 28.3 & moderately fast nova & 2,3 \\
\hline 11. & LMC 1971\#2 & 05 & 40 & 35.22 & -66 & 40 & 35.23 & & poor data & 2 \\
\hline 12. & LMC 1972 & 05 & 28 & 24.66 & -68 & 49 & 42.92 & & poor data & 2 \\
\hline 13. & LMC 1973 & 05 & 15 & 18.58 & -69 & 39 & 46.00 & & poor data & 2 \\
\hline 14. & LMC 1977\#1 & 06 & 05 & 45.50 & -68 & 38 & 12.74 & & poor data & 2 \\
\hline 15. & LMC $1977 \# 2$ & 05 & 05 & 10.87 & -70 & 09 & 01.51 & 20.7 & fast nova & $2,3,6$ \\
\hline 16. & LMC 1978\#1 & 05 & 05 & 52.26 & -65 & 53 & 02.67 & 7.8 & v. fast nova & 2,3 \\
\hline 17 & LMC 1978\#2 & 05 & 00 & 59.65 & -67 & 12 & 44.81 & & poor data & 2 \\
\hline 18. & LMC 1981 & 05 & 32 & 09.27 & -70 & 22 & 11.70 & & spectra indicate v. fast; ONeMg nova & 2,7 \\
\hline 19. & LMC 1987 & 05 & 23 & 50.12 & -70 & 00 & 23.50 & 5.26 & v. fast nova & 2,3 \\
\hline 20. & LMC 1988\#1 & 05 & 35 & 29.33 & -70 & 21 & 29.39 & 39.2 & moderately fast nova & $2,3,8$ \\
\hline 21. & LMC 1988\#2 & 05 & 08 & 01.10 & -68 & 37 & 37.67 & 9.7 & v. fast; ONeMg nova & $2,3,9$ \\
\hline 22. & LMC 1990\#1 & 05 & 23 & 21.82 & -69 & 29 & 48.48 & 7.69 & v. fast; ONeMg nova & $2,3,10$ \\
\hline 23. & LMC 1990\#2 & 05 & 09 & 58.28 & -71 & 39 & 51.49 & 5.26 & Recurrent nova 1968 & \\
\hline 24. & LMC 1991 & 05 & 03 & 44.98 & -70 & 18 & 13.64 & $6 \pm 1$ & v. fast; super bright, low metallicity & 11 \\
\hline 25. & LMC 1992 & 05 & 19 & 19.84 & -68 & 54 & 35.09 & 11.23 & fast nova & 3 \\
\hline 26. & LMC 1995 & 05 & 26 & 50.33 & -70 & 01 & 23.08 & & SS X-ray at late phases. $1.2 M_{\odot} \mathrm{CO} W D$ & 12 \\
\hline 27. & LMC 1997 & 05 & 04 & 26.07 & -67 & 38 & 38.00 & & & \\
\hline 28. & LMC 1999 & 05 & 35 & 32.77 & -69 & 29 & 52.01 & & does not appear to be a nova & \\
\hline 29. & LMC 2000 & 05 & 25 & 01.60 & -70 & 14 & 17.03 & & & \\
\hline 30. & LMC 2002 & 05 & 36 & 46.64 & -71 & 35 & 34.4 & 23 & fast nova & $13,14,15$ \\
\hline
\end{tabular}

${ }^{a}$ References: (1) Buscombe \& de Vaucouleurs (1955), (2) Capaccioli et al. (1990) and references therein, (3) Della Valle \& Livio (1995) and references therein, (4) Sekiguchi et al. (1990), (5) Shore et al. (1991), (6) Canterna \& Thompson (1981), (7) Andrillat \& Dennefeld (1983), (8) Schwarz et al. (1998), (9) Sekiguchi et al. (1989), (10) Vanlandingham et al. (1999), (11) Schwarz et al. (2001), (12) Orio \& Greiner (1999), (13) Liller (2002), (14) Kilmartin \& Gilmore (2002), (15) Gilmore (2002).

The stellar data are corrected for an assumed reddening of $E(V-I)=0.10$ mag. Although the reddening in the LMC has been shown to be varying and clumpy (Udalski et al. 1999), a single value for reddening is assumed here, and is found to be satisfactory while fitting the isochrones to the main-sequence of the CMDs, except in the case of one region, near LMC 1948. Assuming the relation $A_{V}=3.24 E(B-V)$ and $E(V-I)=$ $1.37 E(B-V)$, the value of $A_{V}$ is obtained as $0.355 \mathrm{mag}$. This value of extinction agrees (within errors) with that estimated by Dolphin (2000). Following Pietrzynski \& Udalski (2000) a distance modulus of $18.24 \mathrm{mag}$ for the LMC is assumed here. For this value of the distance modulus, one arcmin on the LMC corresponds to $13.4 \mathrm{pc}$.

The ages of different stellar populations in the vicinity of novae are identified by isochrone fits to the CMDs. An isochrone fit to the MS identifies the age of the youngest population while the isochrone fits to the red giants (RG) identify the ages of the intermediate to old stellar population. The isochrones from Bertelli et al. (1994) with metallicities, $Z=$ 0.008 and $Z=0.004$ are used for the age estimation.

The differential luminosity functions (LFs) of the mainsequence (MS) stars and the red giants are estimated and are used to compare the relative distribution of stars in the CMDs. The LF is estimated by binning the stars in magnitudes, such that the width of each bin is 0.2 mag. The stars bluer than $(V-I)_{0}=0.7$ are assumed to be MS stars and stars redder are assumed to be red giant stars. In order to estimate accurate LFs, the incompleteness in the data should be included. For this, incompleteness in the data presented by Udalski et al. (2000), which is a function of brightness and stellar density are used. Three sets of incompleteness data are available, depending on the stellar density of the field. By visually comparing the stellar density near the location of the novae, the most likely values of data incompleteness for each region are chosen. A limiting magnitude of $V=20.7$ is chosen for the present study. This limiting magnitude implies that the stars in the MS are younger 


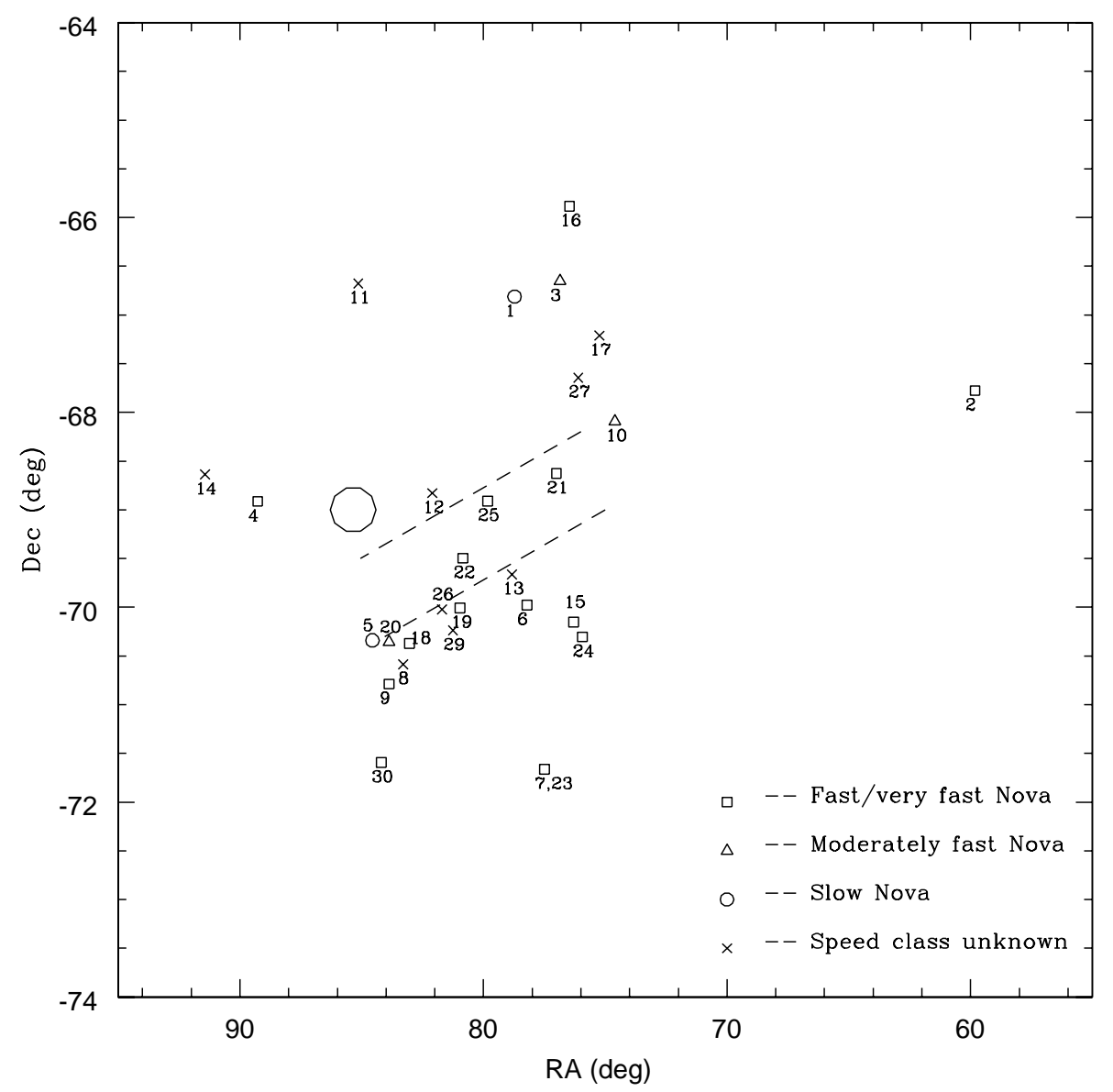

Fig. 1. Distribution of novae across the face of the Large Magellanic Cloud. The Bar and 30 Dor regions are also shown.

than about $1.6 \mathrm{Gyr}$, while the RGB stars are a mixture of both young and old population. This point is to be borne in mind in the discussion of the fraction of stars in the MS and RGB.

\subsubsection{Star clusters}

The star clusters in the vicinity of novae were identified and their properties obtained based on the following catalogs: Pietrzynski et al. (1999: P99), Bica et al. (1999: B99), Bica et al. (1996: B96), Pietrzynski and Udalski (2000: PU2000). B96 present integrated $U B V$ photometry of 624 star clusters and associations in the LMC. They estimate the ages of the clusters based on their integrated colours and hence classify the clusters into SWB types (Searle et al. 1980), which is basically an age sequence. This classification can be used to obtain the approximate age of the clusters. B99 is a revised version of the above catalogue and contains about 1808 star clusters for which the position and extent are tabulated. P99 present photometric data of 745 star clusters and their nearby field, of which 126 are new findings. PU2000 estimate the ages for 600 star clusters presented in the P99 catalogue. The catalogues in B99 and P99 were used to identify the clusters, while B96 and PU2000 were used to estimate the ages of the identified star clusters.

Clusters have been identified within 30 arcmin of the novae using B99. 345 clusters have been identified near 24 novae. Of these, the age estimates for 140 clusters could be obtained from PU2000 and B96. B96 gives the age of the cluster in terms of groups. As the interest is in the overall age of the underlying population rather than the ages of the individual clusters, age groups give a better insight. Therefore, even those clusters whose exact ages are known are also grouped. The number of clusters detected near each nova, the number for which the age is known and the number of clusters in various age groups are tabulated in Table 2. The clusters are grouped into the following five age groups:

(a) clusters with ages $\log \tau<7.5$, indicating clusters of very young age;

(b) clusters with ages $7.5 \leq \log \tau<8.0$, indicating clusters which are relatively young;

(c) clusters with ages $8.0 \leq \log \tau<8.5$, equal to 8.0 and less than 8.5 , indicating a group of moderately young clusters;

(d) clusters with ages $8.5 \leq \log \tau<9.0$;

(e) clusters with ages $\log \tau \geq 9.0$, indicating intermediate population.

Field stars within a radius of a few arcmin, upto a maximum of $10 \operatorname{arcmin}(134 \mathrm{pc})$ around the nova are analysed to study the star formation history, while clusters within 30 arcmin $(\sim 400 \mathrm{pc})$ radius are considered. A larger radius for the clusters is justified as they are being used to study the star formation events which took place on relatively larger scales. 
Table 2. LMC novae: statistics of the star clusters lying within 30 arcmin.

\begin{tabular}{|c|c|c|c|c|c|c|c|}
\hline \multirow[t]{2}{*}{ Nova } & \multirow{2}{*}{$\begin{array}{c}\text { No. of clusters } \\
\text { within } 30 \mathrm{arcmin}\end{array}$} & \multirow{2}{*}{$\begin{array}{l}\text { No. of clusters } \\
\text { with age known }\end{array}$} & \multicolumn{5}{|c|}{ Age groups } \\
\hline & & & $\leq 7.5$ & $7.5-8.0$ & $8.0-8.5$ & $8.5-9.0$ & $\geq 9.0$ \\
\hline \multicolumn{8}{|l|}{ ONeMg novae } \\
\hline LMC 1981 & 15 & 12 & 1 & - & 7 & 3 & 1 \\
\hline LMC 1988\#2 & 9 & 8 & - & 4 & 1 & 2 & 1 \\
\hline LMC 1990\#1 & 17 & 13 & - & 3 & 3 & 3 & 4 \\
\hline \multicolumn{8}{|l|}{ Fast novae } \\
\hline LMC 1937 & 4 & - & - & - & - & - & - \\
\hline LMC 1951 & 24 & - & - & - & - & - & - \\
\hline LMC 1968 & 4 & - & - & - & - & - & - \\
\hline LMC 1970b & 13 & 1 & - & - & 1 & - & - \\
\hline LMC 1977b & 3 & 3 & 1 & - & 2 & - & - \\
\hline LMC 1987 & 24 & 19 & - & 2 & 11 & 4 & 2 \\
\hline LMC 1992 & 5 & 4 & 1 & - & - & 2 & 1 \\
\hline LMC 2002 & 5 & - & - & - & - & - & - \\
\hline \multicolumn{8}{|c|}{ Moderately fast novae } \\
\hline LMC 1936 & 14 & 3 & - & - & 3 & - & - \\
\hline LMC 1971a & 14 & 1 & - & - & 1 & - & - \\
\hline LMC 1988a & 17 & 15 & 1 & 1 & 6 & 5 & 2 \\
\hline \multicolumn{8}{|l|}{ Slow novae } \\
\hline LMC 1926 & 7 & - & - & - & - & - & - \\
\hline LMC 1948 & 37 & 11 & - & 1 & 7 & 3 & - \\
\hline \multicolumn{8}{|c|}{ Speed class unknown } \\
\hline LMC 1970a & 29 & 4 & - & - & 2 & 2 & - \\
\hline LMC 1971b & 10 & - & - & - & - & - & - \\
\hline LMC 1972 & 35 & 3 & 3 & - & - & - & - \\
\hline LMC 1973 & 17 & 9 & 2 & 5 & 2 & - & - \\
\hline LMC 1978b & 8 & 5 & - & 1 & 2 & 2 & - \\
\hline LMC 1995 & 27 & 22 & - & 4 & 11 & 7 & - \\
\hline LMC 1997 & 2 & 2 & - & - & - & 2 & - \\
\hline LMC 2000 & 5 & 5 & - & - & 3 & 1 & 1 \\
\hline
\end{tabular}

\subsection{Results}

In this section, the location and underlying stellar population around individual novae are discussed. Figures 2-16 present the CMDs and the LFs for the regions discussed. The isochrones with their corresponding ages are also shown in the CMDs. The isochrone fits to the CMDs were tried using both the $Z$ values, but $Z=0.008$ isochrones were found to fit the CMDs better. Hence unless otherwise specified, the isochrones referred to in this section are for $Z=0.008$. The fitting of isochrones to the CMDs is based on visual estimation and also there is a gap in the age between two consecutive isochrones. These two introduce some error in the estimation of the age of the stellar population. This error primarily depends on the age of the isochrone considered. The error introduced in the age estimate is of the order of $10 \%$.

Table 3 presents the number of field stars considered around each nova region for which data are available. Column 2 presents the number of stars found in the catalogue and Col. 3 tabulates the total number of stars after applying the incompleteness correction. Also the fraction of stars in the MS, RGB and RGC, estimated as described in Sect. 3.1.1 and applying the incompleteness correction, are tabulated. The CMDs show the number of stars presented in the catalogue, whereas the LF plots show the number of stars after correcting for the data incompleteness. The statistical error at each data point in the LF is estimated and is shown as error bar in the figure.

\subsubsection{Fast novae-ONeMg type}

LMC 1981 This nova is located very close to the Bar, slightly south-east of the Bar. It is one of the 5 novae clustered at this location. Spectroscopy of this nova during outburst indicate it to be of the ONeMg type (Andrillat \& Dennefeld 1983).

This nova has 15 star clusters within 30 arcmin, of which the ages are known for $12.58 \%$ of the clusters in this region have ages between 100-300 Myr, 25\% between 300 Myr and 
Table 3. LMC novae: statistics of field stars in the neighbourhood.

\begin{tabular}{lrrrrrrrr}
\hline \hline Nova & $\begin{array}{r}\text { Observed } \\
\text { stars }\end{array}$ & $\begin{array}{r}\text { Total } \\
\text { stars }\end{array}$ & $\begin{array}{r}\text { MS } \\
\text { stars }\end{array}$ & $\begin{array}{r}\text { Red } \\
\text { giants }\end{array}$ & $\begin{array}{r}\text { MS } \\
\text { fraction }\end{array}$ & $\begin{array}{r}\text { RG } \\
\text { fraction }\end{array}$ & $\begin{array}{r}\text { Clump } \\
\text { stars }\end{array}$ & $\begin{array}{r}\text { Clump } \\
\text { fraction }\end{array}$ \\
\hline ONeMg & & & & & & & & \\
LMC 1981 & 2472 & 2806.1 & 1473.1 & 1333.1 & 0.525 & 0.475 & 639.7 & 0.480 \\
LMC 1988\#2 & 2948 & 3305.6 & 2150.1 & 1155.4 & 0.650 & 0.350 & 589.6 & 0.510 \\
LMC 1990\#1 & 5526 & 9160.9 & 5283.2 & 3877.7 & 0.577 & 0.423 & 1510.1 & 0.389 \\
& & & & & & & & \\
Fast & & & & & & & & \\
LMC 1977\#2 & 2317 & 2605.4 & 1252.6 & 1352.7 & 0.481 & 0.519 & 605.5 & 0.448 \\
LMC 1987 & 4379 & 7158.4 & 3805.1 & 3353.4 & 0.532 & 0.468 & 1429.5 & 0.426 \\
LMC 1992 & 2165 & 3550.6 & 1941.8 & 1608.8 & 0.547 & 0.453 & 712.9 & 0.443 \\
& & & & & & & & \\
Moderately fast & & & & & & & & \\
LMC 1936 & 2157 & 2497.2 & 1606.1 & 891.0 & 0.643 & 0.357 & 466.4 & 0.523 \\
LMC 1988\#1 & 3618 & 4072.0 & 2319.8 & 1752.2 & 0.570 & 0.430 & 915.8 & 0.523 \\
Slow & & & & & & & & \\
LMC 1948 & 2762 & 3115.5 & 1681.4 & 1434.1 & 0.540 & 0.460 & 469.1 & 0.327 \\
Speed class unknown & & & & & & & & \\
LMC 1970\#1 & 3693 & 4176.2 & 2034.3 & 2141.9 & 0.487 & 0.513 & 1045.8 & 0.488 \\
LMC 1973 & 6739 & 10977.6 & 5984.3 & 4993.3 & 0.545 & 0.455 & 2233.0 & 0.447 \\
LMC 1978\#2 & 2160 & 2494.9 & 1540.6 & 954.3 & 0.618 & 0.382 & 467.5 & 0.490 \\
LMC 1995 & 1797 & 2972.2 & 1754.0 & 1218.3 & 0.590 & 0.410 & 523.9 & 0.430 \\
LMC 1997 & 3514 & 4090.9 & 2442.6 & 1648.3 & 0.597 & 0.403 & 869.8 & 0.528 \\
LMC 2000 & 4054 & 6741.1 & 3771.4 & 2969.7 & 0.559 & 0.441 & 1274.6 & 0.429 \\
\hline
\end{tabular}
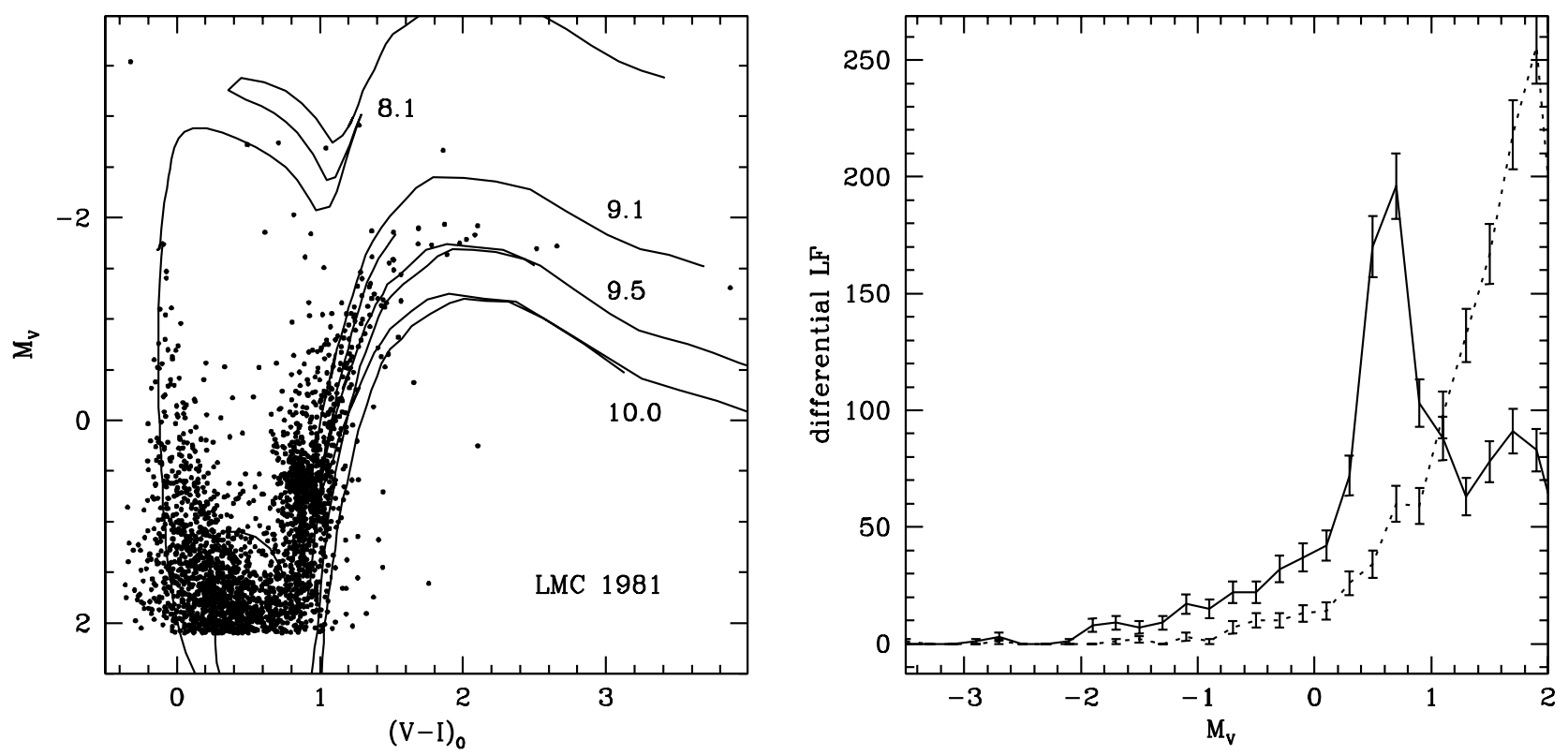

Fig. 2. Left panel: the CMD of 2472 stars within 3 arcmin from LMC 1981. The isochrones fitted to the CMD, with the corresponding value of $\log ($ age) indicated, are also plotted. Right panel: LF of the MS (dotted line) and the red giants (solid line). The error bars indicate the statistical error in the data. 

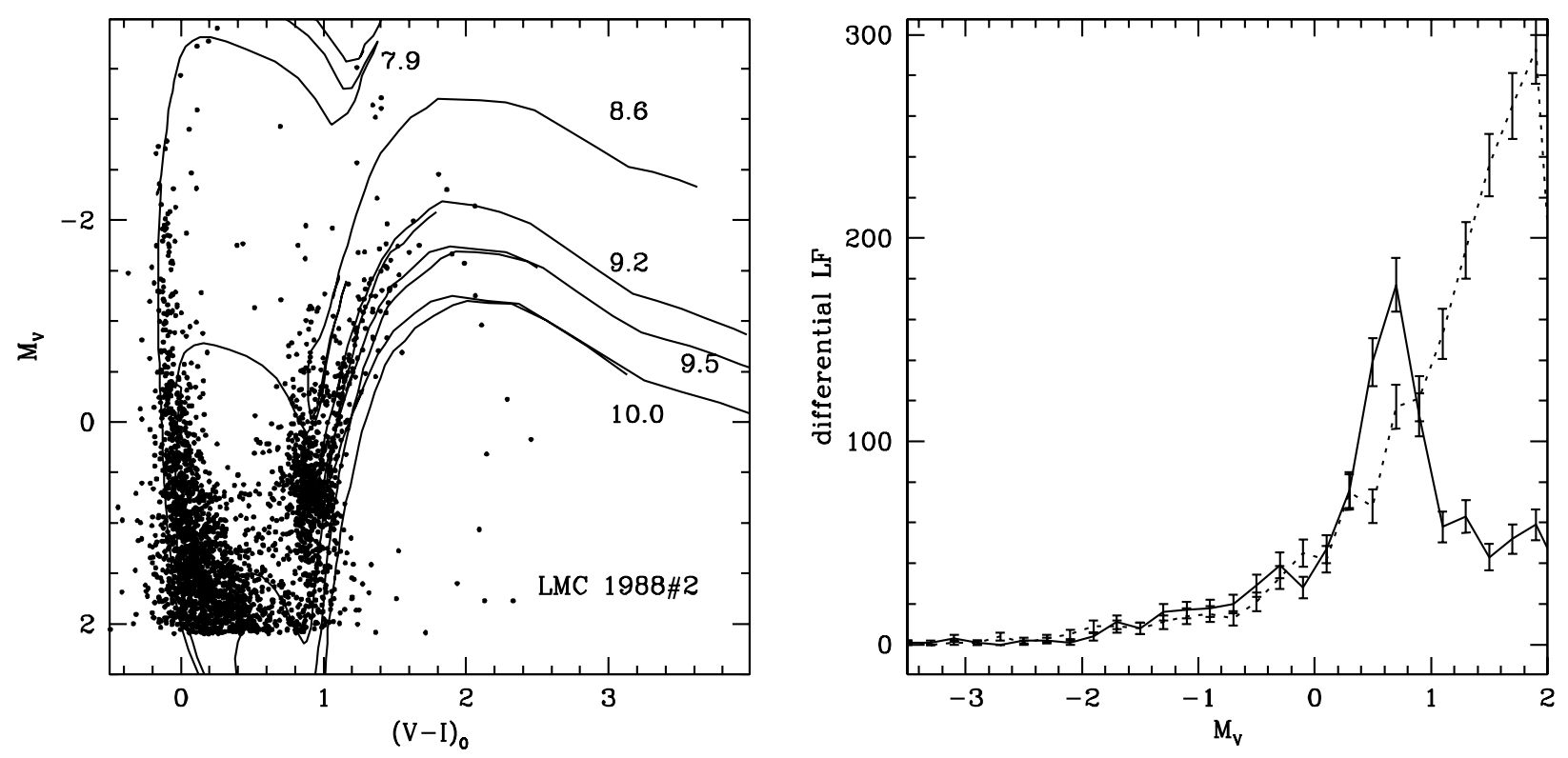

Fig. 3. Left panel: the CMD of 2948 stars within 4 arcmin from LMC 1988\#2. The isochrones fitted to the CMD, with the corresponding value of $\log ($ age $)$ indicated, are also plotted. Right panel: LF of the MS (dotted line) and the red giants (solid line). The error bars indicate the statistical error in the data.

1 Gyr. Only $8 \%$ of clusters are either younger than $30 \mathrm{Myr}$ or older than 1 Gyr. Hence the bulk of cluster formation has occurred in the 100-300 Myr range with a tapering towards 1 Gyr.

The field star population within 3 arcmin radius from the nova location is studied based on a CMD of 2472 stars. The CMD and the LF are plotted in Fig. 2. The CMD shows a well defined RGB, RGC and a MS. The RGB does not show much scatter. Isochrones corresponding to $10 \mathrm{Gyr}, 3.2 \mathrm{Gyr}, 1.3 \mathrm{Gyr}$ and $125 \mathrm{Myr}$ are used to fit the CMD and estimate the ages of the stellar population. These isochrones are also plotted in the figure. A few stars belonging to the $10 \mathrm{Gyr}$ population are seen in the RGB, as indicated by the $10 \mathrm{Gyr}$ isochrone. There seems to be no stellar population between $10 \mathrm{Gyr}$ and $3.2 \mathrm{Gyr}$.

Isochrones of ages $3.2 \mathrm{Gyr}$ and $1.3 \mathrm{Gyr}$ fit the RGB and the RGC as well as the subgiant branch stars which connect the MS and the RGB at the fainter end of the CMD. The stars towards the left side of the $1.3 \mathrm{Gyr}$ isochrone at the RGB belong to a younger population. The brightest stars in the MS are about 125 Myr old.

The RGLF plot shows that the red giant branch is populated evenly with a clear RGC. The RGC seems to taper to the fainter magnitudes. The MSLF shows that the MS does not seem to have very young stars. The upper MS is scantily populated, with a few stars brighter than $M_{V}=0.0$ mag.

The estimated fraction of stars in the CMD reveals that $52.5 \%$ of the stars in this region are in the MS and the rest are in the red giant phase. Among the red giants, $48.0 \%$ are found in the RGC.

LMC 1988\#2 This fast nova is located within projected view of the Bar, towards its north-western side. Spectra presented by Sekiguchi et al. (1989) indicate the nova to be an ONeMg type. One supernova remnant of type Ia is found to be located close to this nova (Williams et al. 1999). 9 star clusters have been identified within 30 arcmin radius, and ages of 8 clusters are known. $50 \%$ of the clusters have ages in the range 30-100 Myr, $12.5 \%$ have ages in the range of 100-300 Myr, $25 \%$ in the range $300 \mathrm{Myr}-1 \mathrm{Gyr}$ and $12.5 \%$ have ages beyond 1 Gyr. This shows that there has been a constant cluster formation, with a rate which doubled during the $300 \mathrm{Myr}-1 \mathrm{Gyr}$ period and quadrupled during 30-100 Myr period.

2948 field stars located within 4 arcmin around the nova were identified. The CMD and the LF of the field stars are plotted in Fig. 3. The CMD shows a very well populated MS with a fair number of young stars. The RGB is well populated with a slightly scattered RGC. The isochrones corresponding to the ages $10 \mathrm{Gyr}, 3.2 \mathrm{Gyr}, 1.6 \mathrm{Gyr}, 400 \mathrm{Myr}$ and $79 \mathrm{Myr}$ are shown in the CMD. The $3.2 \mathrm{Gyr}$ and $1.6 \mathrm{Gyr}$ isochrones fit the bulk of the RGB stars. The CMD shows a population of stars directly above the RGC, which is like the extension of the RGC. These can be fitted with an isochrone of age $400 \mathrm{Myr}$. This is indicative of a star formation event around $400 \mathrm{Myr}$. The brightest stars in the MS are fitted very well with the $79 \mathrm{Myr}$ isochrone, indicating that the star formation stopped around 79 Myr ago.

The RGLF plot shows a well populated RGB with a sharp and strong RGC. The RGB is seen to have a recessing peak towards the fainter magnitudes. The MS is well populated starting from $M_{V}=-2.0 \mathrm{mag}$ and rises gradually beyond $M_{V}=0.0 \mathrm{mag}$.

This region contains $65.0 \%$ of stars in the MS and only $35.0 \%$ stars in the red giants. Among the red giants, $51.0 \%$ of stars are located in the RGC. The relatively high percentage of stars in the MS and the detached MS and RGB at the fainter end of the CMD show that the stars in the MS are predominantly young. 

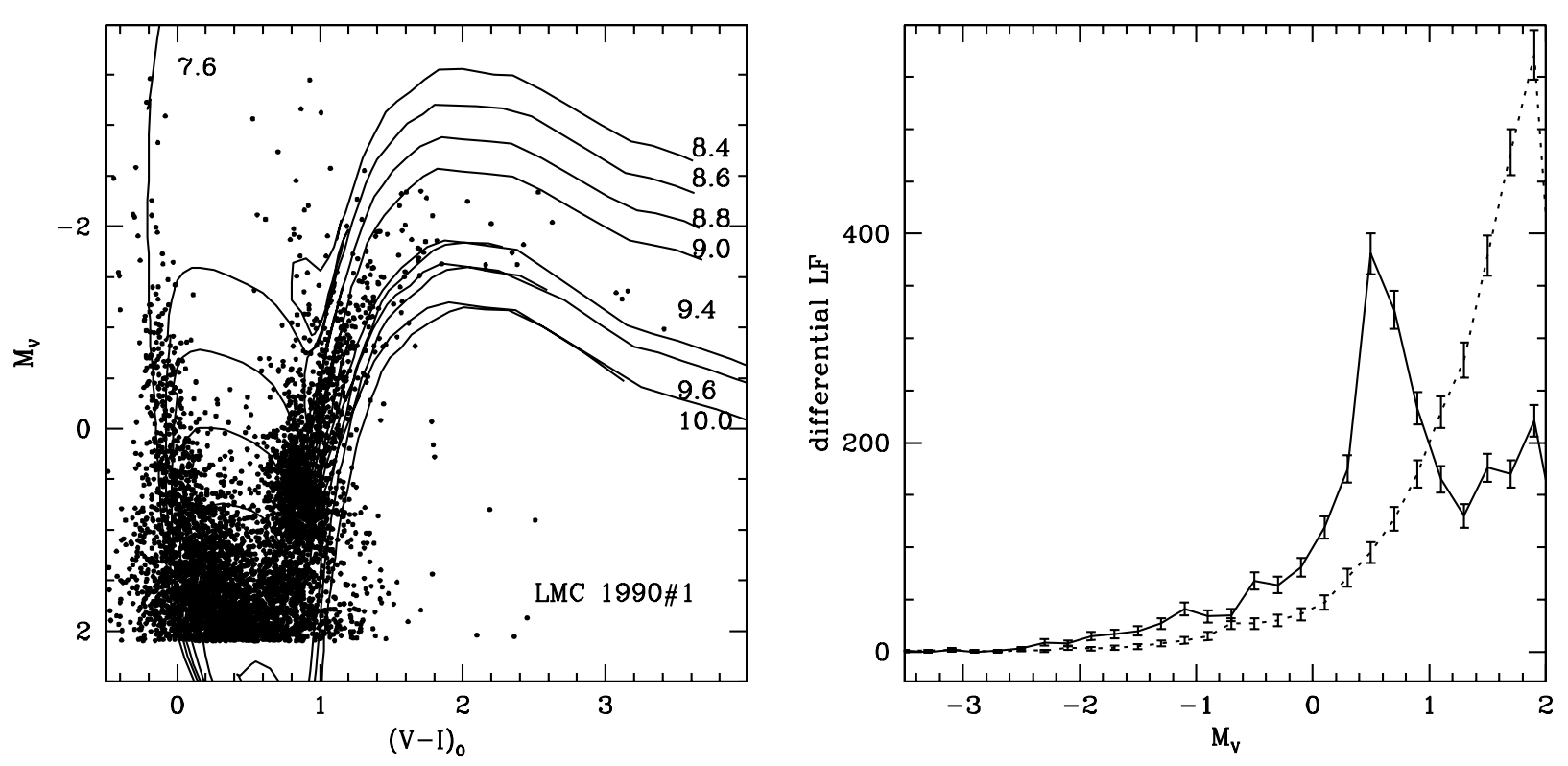

Fig. 4. Left panel: the CMD of 5526 stars within 2 arcmin from LMC 1990\#1. The isochrones fitted to the CMD, with the corresponding value of $\log ($ age $)$ indicated, are also plotted. Right panel: LF of the MS (dotted line) and the red giants (solid line). The error bars indicate the statistical error in the data.

LMC 1990\#1 This ONeMg nova is located in the central region of the projected view of the Bar, but closer to the southern edge. We find that the stellar density in this region is very high. Abundance analyses of the outburst spectra (Vanlandingham et al. 1999) indicate the abundances in the nova material are enhanced in a manner similar to that seen in the Galactic $\mathrm{ONeMg}$ novae.

Seventeen clusters are found in the vicinity, of which the ages are known for 13 clusters. Cluster formation in this region appears to be at a more or less constant rate until $30 \mathrm{Myr}$. $31 \%$ of clusters are with ages more than $1 \mathrm{Gyr}$, and $23 \%$ of clusters in the lower age ranges. Hence the cluster formation rate was lowered around $1 \mathrm{Gyr}$, after an initial higher rate.

The CMD of 5526 field stars within a region of 2 arcmin around the nova are plotted in the left panel of Fig. 4. The RGB, RGC and the MS are well populated. The isochrones corresponding to the ages $10 \mathrm{Gyr}, 4 \mathrm{Gyr}, 2.5 \mathrm{Gyr}, 1 \mathrm{Gyr}, 630 \mathrm{Myr}$, $400 \mathrm{Myr}, 251 \mathrm{Myr}$ and $40 \mathrm{Myr}$ are plotted on the CMD. A few stars are found to belong to the $10 \mathrm{Gyr}$ population, while most of the RGB stars are located between the isochrones of ages $4 \mathrm{Gyr}$ and $250 \mathrm{Myr}$. The isochrone fit to the MS of the CMD shows that the brightest MS stars are $40 \mathrm{Myr}$ old. Both the field stars and the clusters in this region indicate a constant star formation in this region till 30-40 Myr ago.

The RGLF shows that the RGC is less peaked and relatively broader. Also to be noticed are the wings on both sides of the RGC peak in the RGLF. The MSLF is very smooth. The MS contains $57.7 \%$ and RGB has $42.3 \%$ of stars. The shallower peak of the RGC is reflected in the fact that the RGC has only $38.9 \%$ of the total number of red giants. All the above support the suggestion that this region experienced a more or less continuous star formation for a longer duration. This result is consistent with that of Ardeberg et al. (1997) who find a continuous star formation history in the central region of the Bar.

\subsubsection{Fast novae-others}

LMC 1935 This fast nova $\left(t_{3}=25 \mathrm{~d}\right)$ is located about $11^{\circ}$ west of the Bar, in a region devoid of any other novae. No clusters are found near this nova and also no field star data are available.

LMC 1937 This nova is located to the east of the 30 Dor region. Photographic magnitude estimates (Buscombe \& de Vaucouleurs 1955) indicate the nova had a decline rate of $t_{3} \sim 20$ days. There are four star clusters found in the vicinity of this nova. However, the ages of these clusters are not known. No field star data are available in the nova region.

LMC 1951 This extremely fast nova $\left(t_{3}=6.3 \mathrm{~d}\right)$ is located south of the Bar. There are 24 star clusters detected near this nova, but the ages of these clusters are not available. The large number of star clusters near this nova implies it is located in a region where cluster formation has been active.

LMC 1968/1990\#2 This is a recurrent nova of USco type (Sekiguchi et al. 1990; Shore et al. 1991) and is located about 150 arcmin south of the Bar. Recurrent novae of the U Sco type have been identified as systems which could be evolving towards supernovae of type Ia (e.g. Starrfield et al. 1985; Hachisu et al. 2000). There are 4 star clusters located near this nova, but their age estimates are unavailable.

LMC 1970\#2 This nova is one among the 5 novae clustered at the south-east end of the Bar. There is only one star cluster near this nova and the age estimate is not available.

LMC 1977\#2 This nova is located to the south of the Bar, and also very close to LMC 1991, which is also a fast nova. 

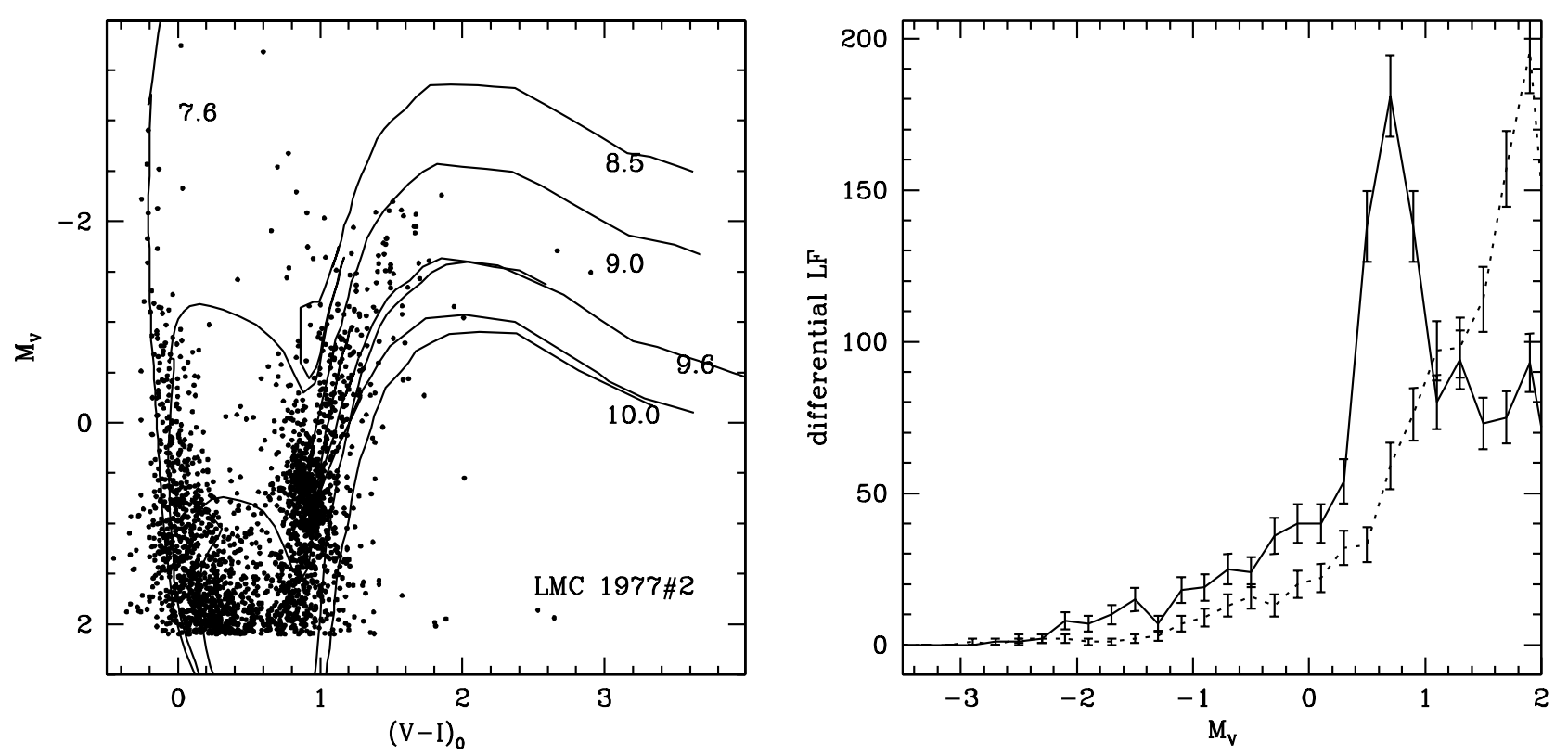

Fig. 5. Left panel: the CMD of 2317 stars from a nearby region within 10 arcmin from nova LMC 1977\#2. The isochrones fitted to the CMD, with the corresponding value of $\log ($ age) indicated, are also plotted. Right panel: LF of the MS (dotted line) and the red giants (solid line). The error bars in the figure indicate the statistical error in the data.

Spectroscopic development during outburst was similar to Galactic novae (Canterna \& Thompson 1981). There are only three star clusters near the nova. One cluster is younger than $30 \mathrm{Myr}$ and two clusters are in the range 100-300 Myr. The fact that only three clusters are found in the neighbourhood indicates that this region may not have experienced high rates of cluster formation.

The CMD of 2317 nearby field stars is plotted in Fig. 5. It may be noted here that the field being considered is not centered around the nova, but is within 10 arcmin of the nova. Most of the RGB stars are populated in the age range $4 \mathrm{Gyr}$ to $1 \mathrm{Gyr}$, with a few belonging to the $10 \mathrm{Gyr}$ population. The MS and the RGB are well disconnected even at fainter magnitudes. The MS is well populated and wide upto $M_{V}=-1.0 \mathrm{mag}$, above which, only a few bright stars are seen. These indicate a $300 \mathrm{Myr}$ old population as estimated by the isochrone fit. The isochrone fit to the brightest stars in the MS indicates these stars are 40 Myr old.

The RGC is found to have a sub clump towards the fainter end. This feature is more clearly seen in the RGLF plot, which is indicated by a second peak.

This region has lesser fraction of stars in the MS, with $48.1 \%$ of stars in the MS and the rest $51.9 \%$ in the RGB. In fact, this is one of the two regions, where the percentage of stars in MS is less than that in the RGB. The RGC contains $44.8 \%$ of the stars in the RGB. It appears that this region had a continuous star formation between 4.0-1 Gyr ago, with most of the stars probably having ages closer to the lower age limit, and this did not result in the formation of star clusters. Another event of star formation took place around $300 \mathrm{Myr}$, resulting in the formation of the two clusters, along with some of the field stars. Then, later, around 30-40 Myr, there was another star formation, which managed to form one cluster. It is suggested that the lesser fraction of stars in the MS is due to a combination of (a) a major fraction of stars being formed in the star formation event before $\sim 1 \mathrm{Gyr}$ and (b) absence of star formation between $1 \mathrm{Gyr}$ and $300 \mathrm{Myr}$.

LMC 1978\#1 This extremely fast nova $\left(t_{3} \sim 8 \mathrm{~d}\right)$ is located north of the Bar. In fact, this is the northern most nova found in the LMC. No star clusters are found near this nova.

LMC 1987 This extremely fast nova is located just below the Bar, in a region that has produced two more novae; LMC 1995 and LMC 2000. There are 24 star clusters in the vicinity and ages are known for 19 of them. This indicates that this region had active cluster formation. The maximum number of star clusters were formed during the period 100-300 Myr, when $58 \%$ of the clusters were formed. $21 \%$ of clusters were formed during the $300 \mathrm{Myr}-1 \mathrm{Gyr}$ period, $10 \%$ formed during the 30-100 Myr period and the rest during the period before $1 \mathrm{Gyr}$. It appears that this region has been forming clusters from the beginning, with an increase in the rate within the last $1 \mathrm{Gyr}$, which increased further between 300-100 Myr. Subsequently the rate appears to have reduced and stopped by $30 \mathrm{Myr}$. This in effect implies a continuous cluster formation till $30 \mathrm{Myr}$.

The CMD and LF of stars within 2 arcmin radius of the nova are plotted in Fig. 6. The isochrone fit reveals that the the stars in the RGB have ages in the range 1.3-3.2 Gyr. Very few stars belonging to the age of $10 \mathrm{Gyr}$ is seen in the CMD. The broad MS below $M_{V}=0.0$ mag indicates a continued star formation from about $1.3 \mathrm{Gyr}$ to lesser ages, probably till $300 \mathrm{Myr}$. The brightest part of the MS is found to be $63 \mathrm{Myr}$ old. There are a few bright red giants, which appear to be slightly older than 63 Myr. The cluster formation and star formation appear to be well correlated in this region. 

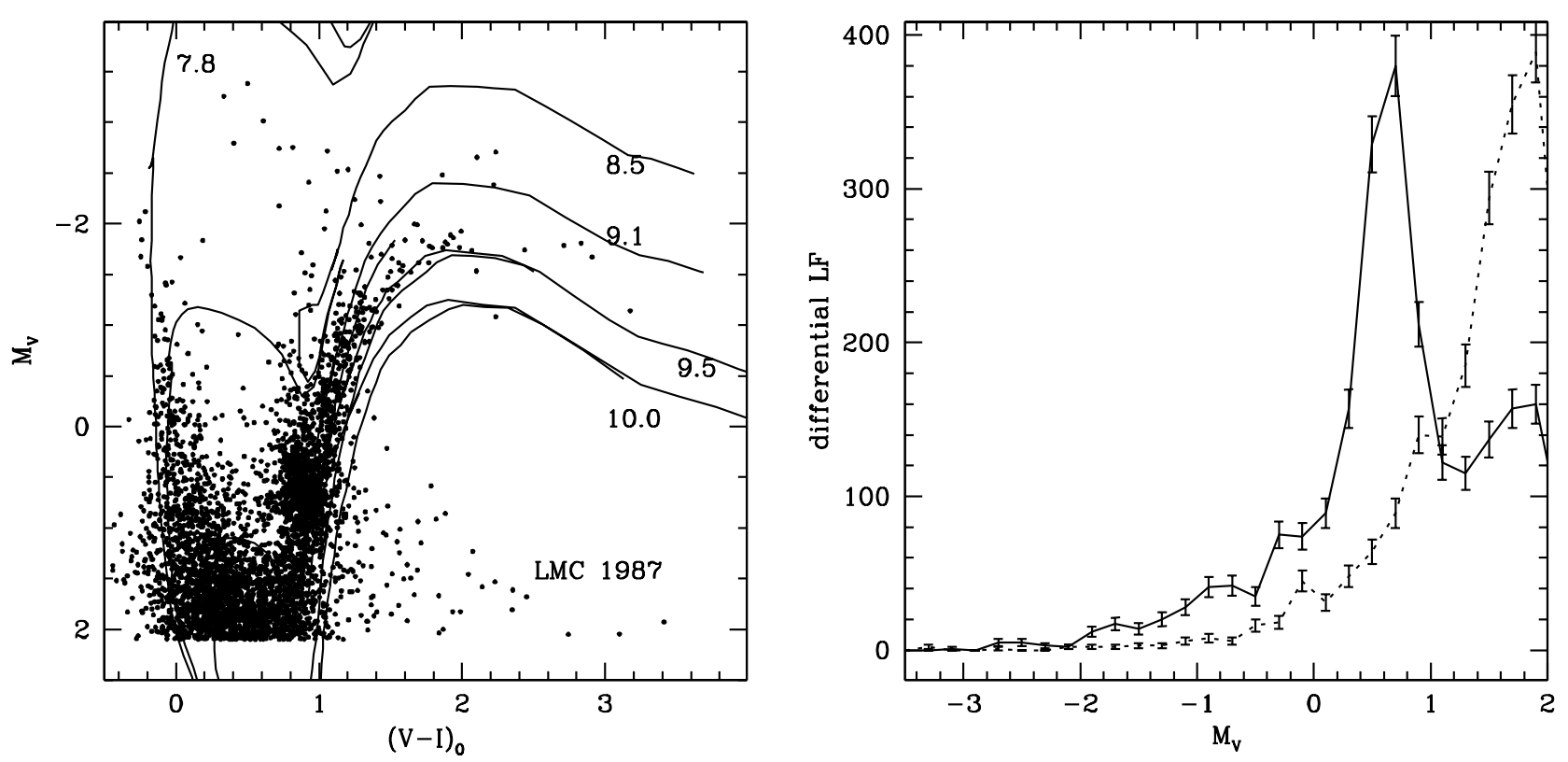

Fig. 6. Left panel: the CMD of 4379 stars within 2 arcmin from LMC 1987. The isochrones fitted to the CMD, with the corresponding value of $\log (\mathrm{age})$ indicated, are also plotted. Right panel: LF of the MS (dotted line) and the red giants (solid line). The error bars indicate the statistical error in the data.

The RGLF shows that the RGB is well populated, indicating the presence of bright red giants. The RGC has a slanting peak. Amongst the total number of stars, $53.2 \%$ are in the MS and the rest $46.8 \%$ in the RGB. Further, $42.6 \%$ of the stars in the RGB are found in the RGC.

LMC 1991 This extremely fast nova is located to the south of another fast nova, LMC 1977\#2. LMC 1991 was detected as a super bright nova with very low metallicity in the ejected material (Della Valle 1991; Schwarz et al. 2001). There are 3 very young star clusters within 10 arcmin radius from the nova location.

LMC 1992 This fast nova is also located in the Bar region. One type Ia supernova remnant is located close to the nova. There are five star clusters located near the nova and ages of four have been estimated. One cluster is with age less than $30 \mathrm{Myr}$. Two clusters are in the age range $300 \mathrm{Myr}-1 \mathrm{Gyr}$ and one cluster is older than $1 \mathrm{Gyr}$. The ages and numbers of the clusters indicate that this region did not experience much cluster formation. The presence of the young cluster indicates that there has been a recent episode of cluster formation.

The CMD and LF of 2165 stars located within 4 arcmin of the nova, is shown in Fig. 7. The CMD indicates an absence of stars belonging to the old population. The reddest stars in the RGB have an age of $2.5 \mathrm{Gyr}$, as seen from the isochrone fit. The star formation in this region seems to have continued till about $500 \mathrm{Myr}$, as indicated by the corresponding isochrone fit and the broad MS below this isochrone. The MS is not so well populated in the brighter end. The isochrone fit indicates that the brightest stars are $63 \mathrm{Myr}$ old. There is no evidence for a strong population between the ages $500 \mathrm{Myr}$ and $63 \mathrm{Myr}$. The star formation history presented by the field stars is very similar to the cluster formation history, except that no counter part for the youngest star cluster is seen.

The RGLF shows a clear RGC with a flat peak. The RGC profile tapers towards the fainter magnitudes. The MSLF shows that there are very little stars brighter than $M_{v}=-1.0$ mag and the LF rises after that. $54.7 \%$ of stars are located in the MS, and the rest $45.3 \%$ are in the RGB. The RGC has $44.3 \%$ of the RGB stars. Star formation appears to have started a little later in this region. This region appears to be dominated by intermediate age stars and stars aged around $500 \mathrm{Myr}$.

LMC 2002 This nova was discovered by Liller (2002) on 3.1 March 2002, at a photographic magnitude of $m_{\mathrm{pg}}=10.5$. A low dispersion spectrum showed strong $\mathrm{H} \alpha$ and $\mathrm{H} \beta$ emission lines confirming its identification as a nova. Subsequent photometry (Kilmartin \& Gilmore 2002; Gilmore 2002) indicate a decline rate of $\sim 0.13 \mathrm{mag} / \mathrm{day}$ ( $t_{3} \sim 23$ days), placing this nova amongst the fast category. Five star clusters are found near this nova, but age estimates for none of these clusters are available. Also, no field star data are available.

\subsubsection{Moderately fast novae}

LMC 1936 This nova is located to the north, about 75 arcmin from the Bar. There are 14 star clusters found near the nova, of which ages are available only for 3 . All three star clusters have ages in the range 100-300 Myr. The high number of star clusters indicates that cluster formation has been quite active in this region.

The CMD of the 2157 stars belonging to the nearby region, 10 arcmin away from the nova, is presented in Fig. 8. The CMD shows that the RGB is not very well populated. The $2.0 \mathrm{Gyr}$, $1 \mathrm{Gyr}, 300 \mathrm{Myr}$ and $63 \mathrm{Myr}$ isochrone fits to the CMD are 

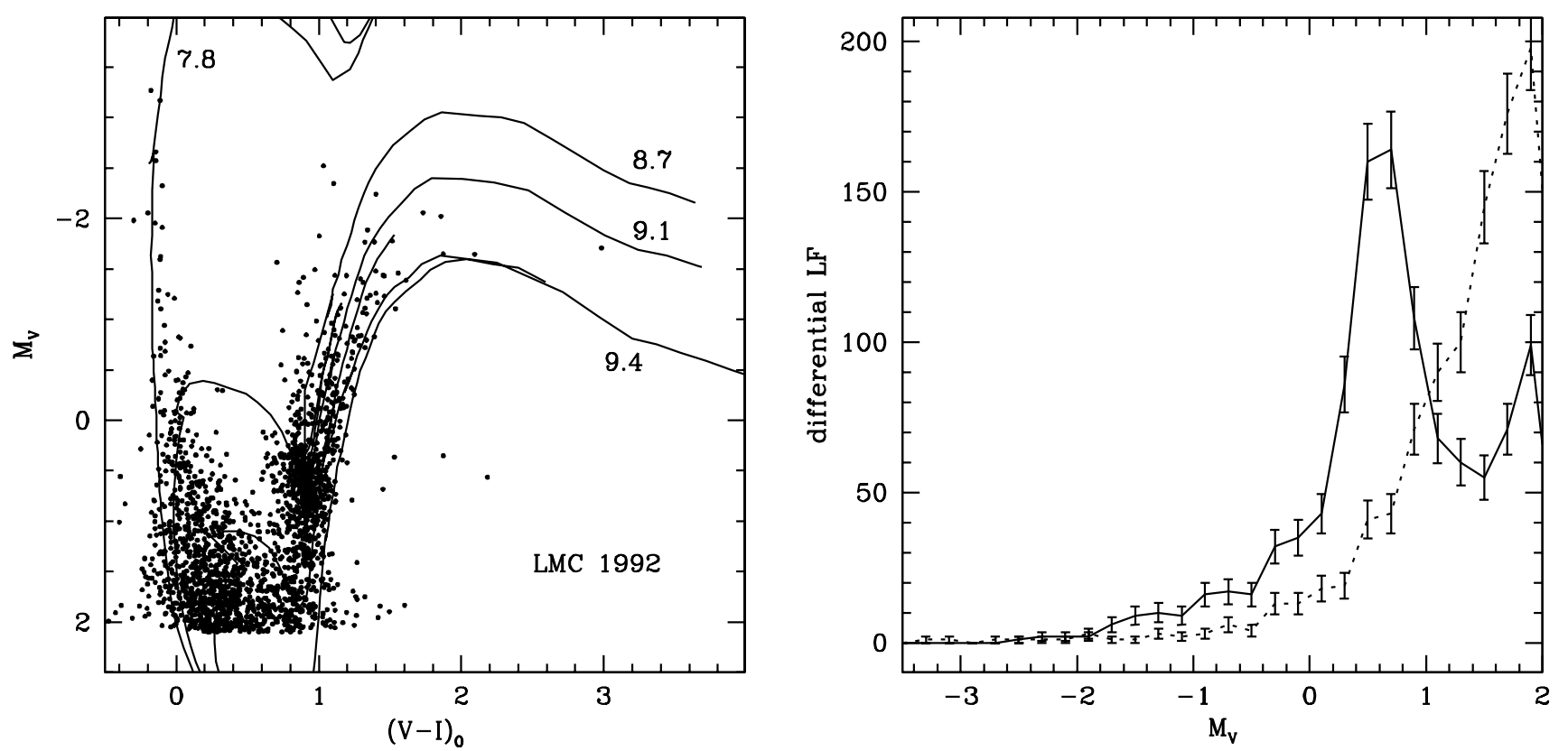

Fig. 7. Left panel: the CMD of 2165 stars within 4 arcmin from LMC 1992. The isochrones fitted to the CMD, with the corresponding value of $\log ($ age) indicated, are also plotted. Right panel: LF of the MS (dotted line) and the red giants (solid line). The error bars indicate the statistical error in the data.
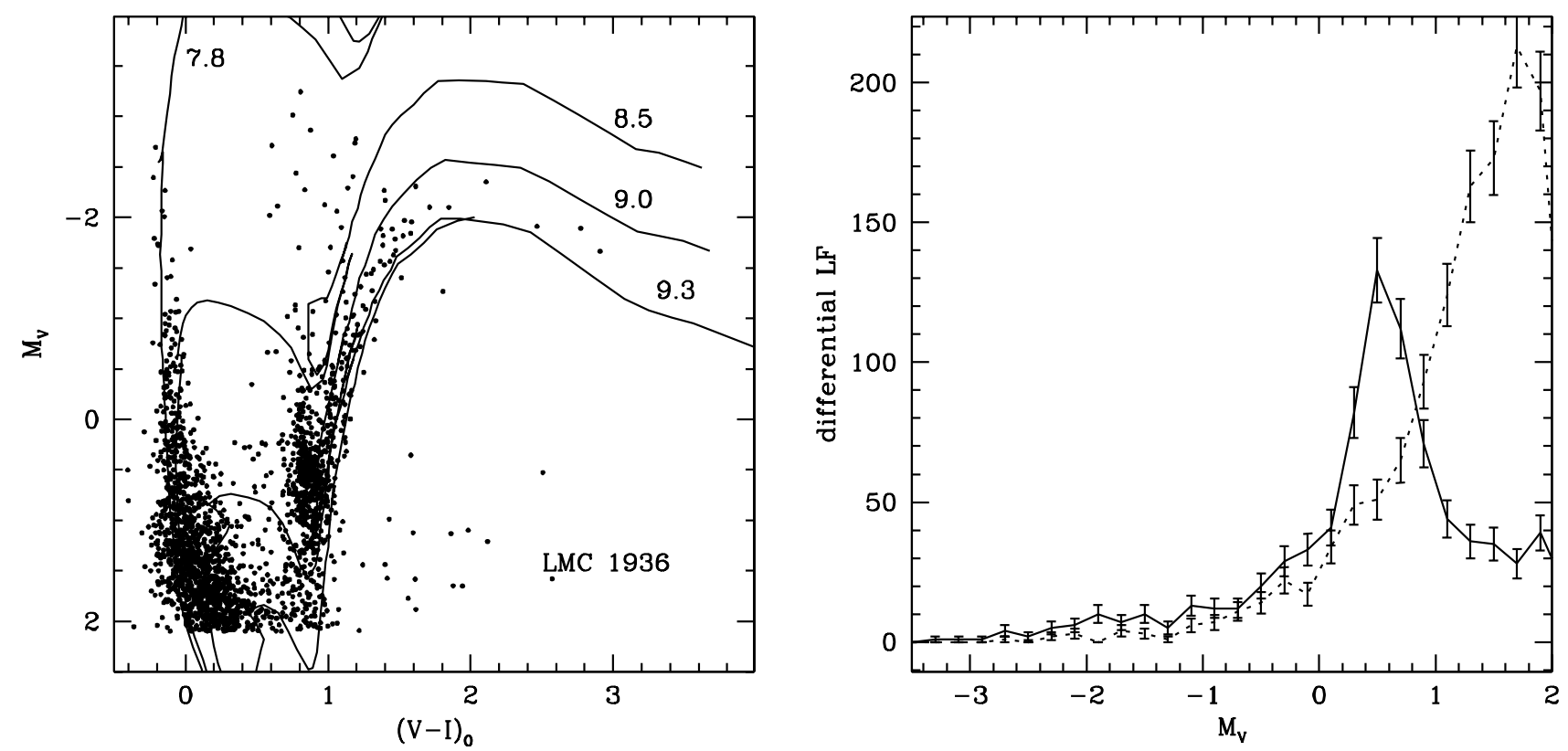

Fig. 8. Left panel: the CMD of 2157 stars from a nearby region within 10 arcmin from nova LMC 1936. The isochrones fitted to the CMD, with the corresponding value of $\log ($ age) indicated, are also plotted. Right panel: LF of the MS (dotted line) and the red giants (solid line). The error bars show the statistical error in the data.

shown in the figure. Stars belonging to the old population are not found in the CMD. Also the reddest stars in the RGB are only $2.0 \mathrm{Gyr}$ old, as indicated by the isochrone fit. If $Z=0.004$ isochrones are used, then the reddest stars in the CMD are found to be $3.2 \mathrm{Gyr}$ old. As we do not have an independent estimate of the metallicity and there is no indication of reduced metallicity in this region, the $Z=0.008$ isochrones are adopted and the age of the oldest stars is estimated to be $2.0 \mathrm{Gyr}$.
The MS and the RGB are well separated at the fainter levels and also show that the RGB is very meagerly populated while the MS is well populated. The MS shows a sudden decrease in the number of stars brighter than $M_{V}=-1.0 \mathrm{mag}$. This indicates another star formation event, and the isochrone fit shows that this happened $300 \mathrm{Myr}$ ago. This coincides with the formation of the star clusters around this region. The brightest part of the MS is estimated to have an age of $63 \mathrm{Myr}$. 

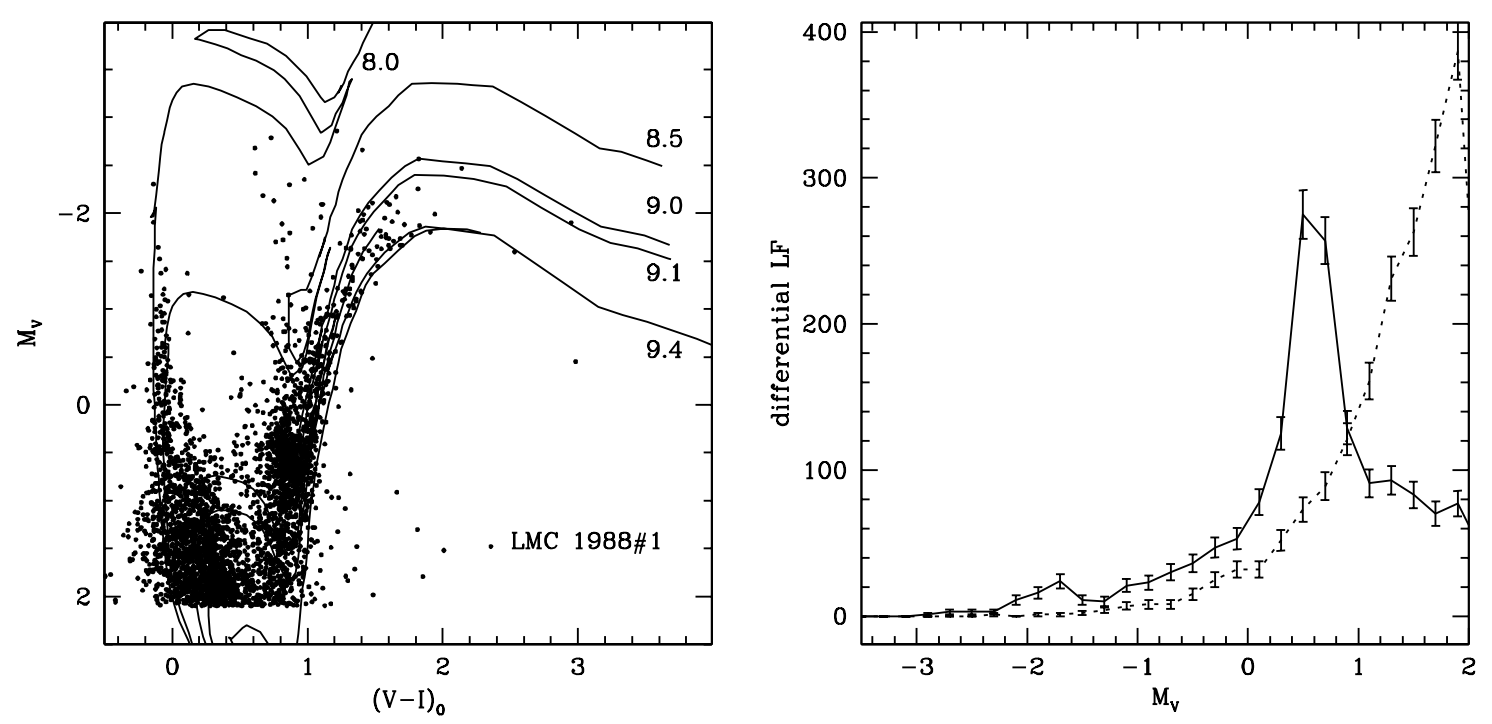

Fig. 9. Left panel: the CMD of 3618 stars within 2 arcmin from LMC 1988\#1. The isochrones fitted to the CMD, with the corresponding value of $\log ($ age $)$ indicated, are also plotted. Right panel: LF of the MS (dotted line) and the red giants (solid line). The error bars indicate the statistical error in the data.

The RGLF plot shows that the RGC has a ramping region on both sides of the peak. The RGC contains $52.3 \%$ of stars in the RGB. This region also has a high population of MS stars (64.3\%) when compared to the RGB stars, similar to the region surrounding LMC 1988\#2.

LMC 1971\#1 This nova is located to the west of the Bar and just outside the Bar. There are 14 star clusters found near the nova, of which the age of only one cluster is known. The age of the cluster falls in the 100-300 Myr range. This region has undergone a fair amount of cluster formation, similar to LMC 1936. No field star data are available for this region.

LMC 1988\#1 This nova is located at the south-east edge of the Bar and is one of the five clustered novae found in that location. The UV, optical and infrared outburst data indicate this nova to be a CO, optically thin dust-forming nova (Schwarz et al. 1998). There are 17 star clusters detected near the nova and ages are known for 15 of them. Forty percent of the clusters have ages in the range $100-300 \mathrm{Myr}, 33.3 \%$ of clusters have ages in the range $300 \mathrm{Myr}-1 \mathrm{Gyr}, 13.3 \%$ clusters are older then $1 \mathrm{Gyr}$. This region thus appears to have experienced a 2.5 times enhancement in the formation of clusters, between $300 \mathrm{Myr}-1 \mathrm{Gyr}$, and increased a little more around $300 \mathrm{Myr}$ and continued till $100 \mathrm{Myr}$. In the last $100 \mathrm{Myr}$, this region has formed 1 cluster older than $30 \mathrm{Myr}$ and one cluster younger than 30 Myr. Hence there has been a continuous formation of clusters with varying rates.

The CMD of 3618 field stars within a radius of 2 arcmin from the nova is shown in Fig. 9. A broad RGB, a prominent clump and the presence of a few bright giants are seen in the evolved part of the CMD. Isochrone fit to the right most end of the RGB indicates that the oldest stars are $2.5 \mathrm{Gyr}$ old. The stars belonging to a population older than $2.5 \mathrm{Gyr}$ is not found. At the fainter of the CMD, the MS and the RGB are not well separated, but joined together due to the presence of subgiants. The MS is wide upto $M_{V}=0.5 \mathrm{mag}$ corresponding to the turnoff point of the $1 \mathrm{Gyr}$ isochrone. The width of the MS decreases at brighter magnitudes, indicating a probable decrease in the star formation. The isochrone of age $300 \mathrm{Myr}$ shows that the star formation probably continued until then. The vertical extension of the RGC, as seen in the CMD also indicates that stars younger than $1 \mathrm{Gyr}$, in the range $500 \mathrm{Myr}-1 \mathrm{Gyr}$, are present. The brightest part of the CMD is found to be $100 \mathrm{Myr}$ old. There is no indication of the presence of stars younger than 100 Myr.

The RGLF indicates some excess stars at the bright end of the RGB, which is a combined effect of the old red giants and the bright and young red giants. The RGC is seen to have a broad wing on the brighter side, probably due to the contribution of stars around $1 \mathrm{Gyr}$ and younger. The MSLF shows an evenly populated MS which rises gradually.

The MS has $57.0 \%$ of stars and the rest $43.0 \%$ of stars are in RGB. The RGC has $52.3 \%$ of the RGB stars. It is striking to note that the regions around both the moderately fast novae have the same fraction of RGB stars in the RGC. The fairly high percent of stars in the MS indicates active star formation in the last few hundred million years. The star clusters and the field stars present a more or less similar star formation history until the last 100 Myr. However, while there are no field stars younger than $100 \mathrm{Myr}$, one star cluster younger than $100 \mathrm{Myr}$ is found in this region.

\subsubsection{Slow novae}

LMC 1926 This nova is located to the north of the Bar and to the east of the moderately fast LMC 1936. A supernova remnant of type Ia has been detected about half a degree south of the nova. There are 7 star clusters found in the vicinity of the nova, but ages of none of the clusters are known. 

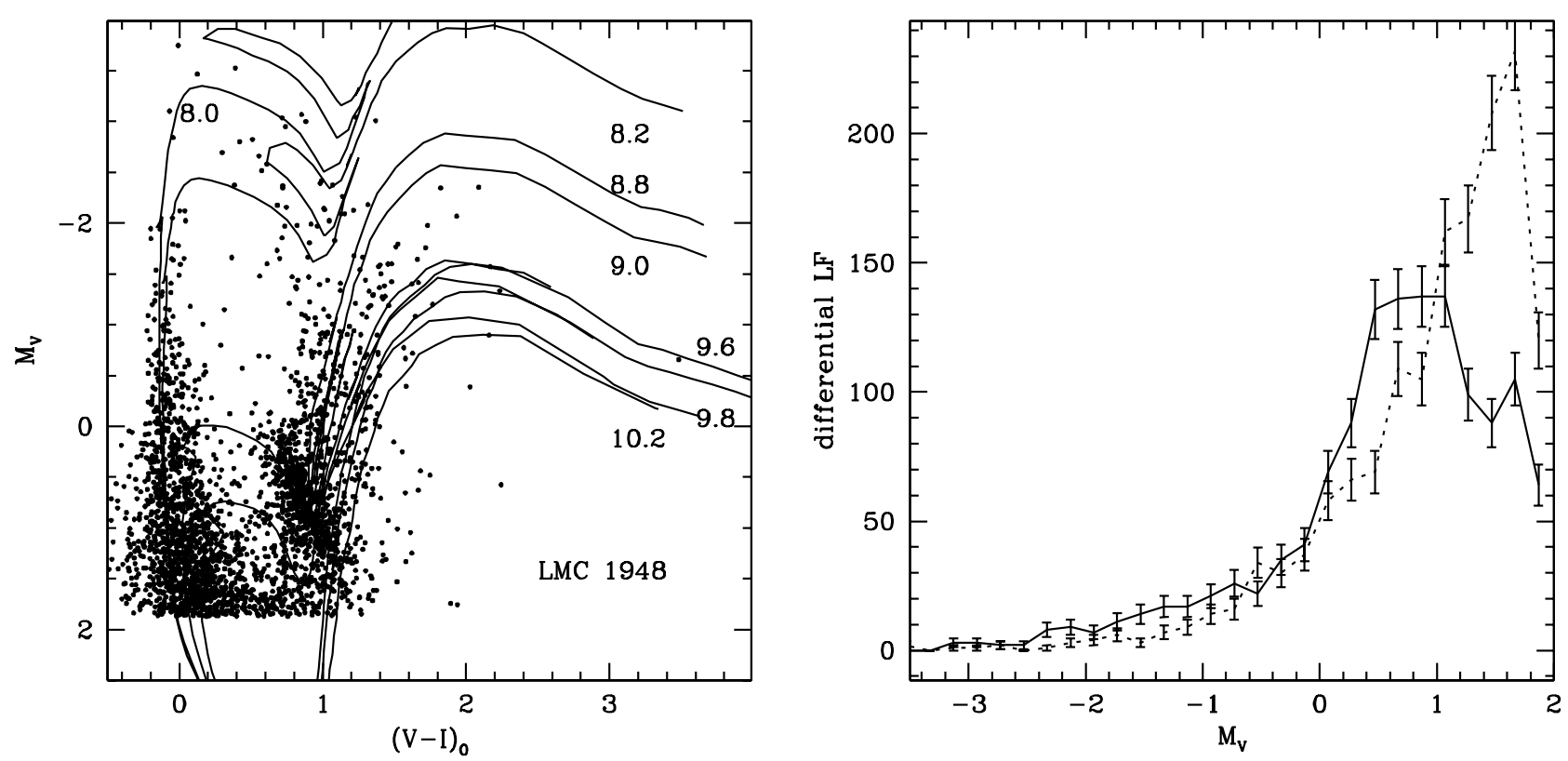

Fig. 10. Left panel: the CMD of 2762 stars within 2 arcmin from LMC 1948. The isochrones fitted to the CMD, with the corresponding value of $\log ($ age) indicated, are also plotted. Right panel: LF of the MS (dotted line) and the red giants (solid line). The error bars indicate the statistical error in the data.

LMC 1948 This is the only slow nova found near the Bar region. This nova also belongs to the cluster of novae near the south-east edge of the Bar. 37 star clusters are found in the vicinity of the nova, of which ages are known for 11 of them. None of the clusters are found with age more than 1 Gyr. $27 \%$ are within the age range $300 \mathrm{Myr}-1 \mathrm{Gyr}, 63.6 \%$ are in the age range 100-300 Myr and 9\% have ages less than $100 \mathrm{Myr}$, but more than 30 Myr. Hence the cluster forming activity peaked during 100-300 Myr, with clusters forming with lesser efficiencies on both sides of this age range.

The CMD of 2762 field stars within a radius of 2 arcmin from the nova is shown in Fig. 10. It appears that this region has a higher reddening, and a value of $E(V-I)=0.25 \mathrm{mag}$ is adopted. The resultant CMD indicates that the RGB and the RGC are wide, which could be due to differential reddening in the region. However, a close look at the width of the MS indicates that it is not too different from the other regions studied in this work. It thus appears that the differential reddening is not too large and the width of the RGB and the RGC is real. Extremely red stars are seen in the RGB, indicative of the presence of very old stars. It is found that a $10 \mathrm{Gyr}$ isochrone fits most of the red stars. It is also likely that stars older than $10 \mathrm{Gyr}$ could be present in this region. The isochrones shown in the CMD are $10 \mathrm{Gyr}, 6.3 \mathrm{Gyr}, 4 \mathrm{Gyr}, 1 \mathrm{Gyr}, 630 \mathrm{Myr}, 160 \mathrm{Myr}$ and 100 Myr. It can be seen that the RGB population has the widest range of ages, from $10 \mathrm{Gyr}$ to $630 \mathrm{Myr}$. Also, the RGB stars are evenly populated within this age range, with the $4 \mathrm{Gyr}$ isochrone falling midway. A simple, visual estimate indicates that approximately half the RGB population is older than $4 \mathrm{Gyr}$. At the fainter end of the CMD, the RGB and the MS are fairly separated with the MS well populated, while the RGB is scantily populated. At around $630 \mathrm{Myr}$, a turn-off near the MS is seen, indicating a star formation event, which could have resulted in the formation of a few star clusters. Star formation might have continued further. A well populated MS as seen in the CMD also indicates the same. The brightest stars in the CMD are found to be $100 \mathrm{Myr}$ old. The bright red giants are found to be 160 Myr old.

The RGLF profile in this region is quite interesting. It shows a very flat RGC, quite unlike the RGC seen in the other regions. The prominent peak of the RGC, which is seen in the other regions is missing, indicating a marked difference in the star formation history, for ages beyond $1 \mathrm{Gyr}$. The RGLF rises initially showing the presence of a good number of bright red giants. Another important point to be noted is that at the fainter end of the RGC profile, which is a contribution from older stars, the fraction of stars is relatively higher.

The MS has $54.0 \%$ of the stars and RGB has $46.0 \%$ of the total stars found in this region. Also, the RGC has only $32.7 \%$ of the stars in the RGB. This value is very small compared to the other regions. This indicates that the star formation occurred in a continuous fashion for a duration much longer than in other regions.

A point of particular interest to note is the significant presence of an old star population, up to an age of about 10 Gyr. This population is absent in the regions of other novae in the Bar region. This point is of particular significance as LMC 1948 is the only slow nova in this region. All other novae detected near the Bar region are either fast or moderately fast.

\subsubsection{Others}

The stellar population around the locations of novae for which the speed class is not available are discussed in this section.

LMC 1970\#1 This nova is located near the south-east edge of the Bar. This is one among the five novae clustered in this 

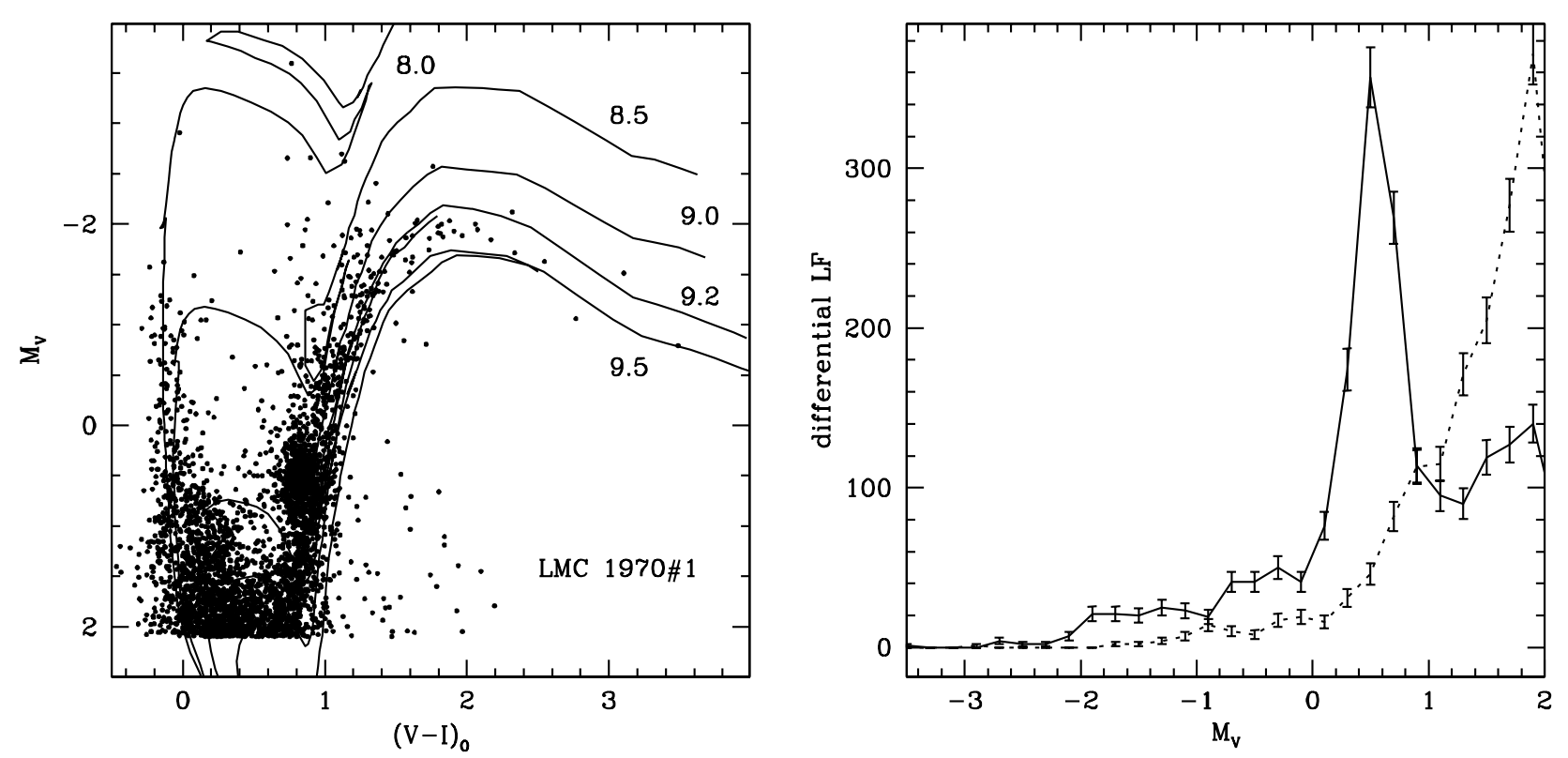

Fig. 11. Left panel: the CMD of 3693 stars within 5 arcmin from LMC 1970\#1. The isochrones fitted to the CMD, with the corresponding value of $\log ($ age) indicated, are also plotted. Right panel: LF of the MS (dotted line) and the red giants (solid line). The error bars indicate the statistical error in the data.

region. 29 star clusters are found around this nova, of which ages are known for only 4. Two clusters are in the age range 100-300 Myr and the other two in the 300-1 Gyr range. The large number of clusters indicate that there has been a significant amount of cluster formation in this region.

The CMD of 3693 stars within a radius of 5 arcmin of the nova is shown in Fig. 11. Isochrones of ages 3.2 Gyr, 1.6 Gyr and 1 Gyr are fitted to the RGB. There is no clear signature of stellar population older than 3.2 Gyr. This star formation continued till about $1 \mathrm{Gyr}$, as implied by the reduction in the width of the MS above the $1 \mathrm{Gyr}$ isochrone. The wider part of the MS at $M_{V}=-1.0$ and the bright red giants indicate that the star formation continued, probably till $315 \mathrm{Myr}$. The brightest stars in the CMD are found to be 100 Myr old.

The RGC is a narrow and tall peak, indicative of a strong star formation event. The MSLF shows that the profile rises slowly. This region is one of the two regions, other than the region around LMC 1977\#2, with lesser fraction of stars in the MS, than in the RGB. This region has $48.7 \%$ stars in the MS. The RGC has $48.8 \%$ of the stars in the RGB.

LMC 1971\#2 This nova is located to the north of the Bar and 30 Dor. This is an isolated nova, away from the region where most novae are detected. There are 10 star clusters near the nova and the ages are not known for any cluster. No field star data are available.

LMC 1972 This nova is located very close to the projected location of the Bar, towards the north. There are 35 star clusters around the nova. Ages of three clusters are known and they are all younger than $30 \mathrm{Myr}$. The large number of clusters and the presence of very young clusters indicate that the cluster formation has been very vigorous in this region. Field star data are not available for this region.
LMC 1973 This nova is located very close to the Bar region, on its southern side. There are 17 star clusters near this nova, of which ages are known for 9 star clusters. $55.6 \%$ of the clusters have ages in the range $30-100 \mathrm{Myr} .22 .2 \%$ of star clusters have ages less than $30 \mathrm{Myr}$ and $22.2 \%$ have ages between 100-300 Myr. From the cluster data it is seen that this region had an active cluster formation event in the recent past, that is $30-100 \mathrm{Myr}$, with a little subsided activity before and after that. As there are 17 clusters, it is more or less clear that this region has had strong cluster formation episodes.

The CMD of 6739 field stars which lie within a radius of 3 arcmin from the nova is shown in Fig. 12. The isochrones of ages 4 Gyr, $800 \mathrm{Myr}$ and $63 \mathrm{Myr}$ are shown in the CMD. The merged MS and RGB at the fainter end of the CMD shows that the star formation was more or less continuous between 4 Gyr and 800 Myr. The CMD indicates this region has a small fraction of an old population of around 10 Gyr. Subsequently, perhaps around $4 \mathrm{Gyr}$, star formation started vigorously and continued till $800 \mathrm{Myr}$, following which, until 63 Myr back, star formation occurred in a more subdued manner.

The RGLF shows a smooth curve for the RGB and a not so narrow peak for the RGC. The smoothly rising RGB profile indicates that star formation has been more or less continuous. The MS is also a gradually rising smooth profile. This region has $54.5 \%$ of stars in the MS and RGC has $44.7 \%$ of the RGB stars.

LMC 1977\#1 This nova is located about $5^{\circ}$ north-east of the Bar and is the eastern most nova detected in the LMC. The fast nova LM 1937 is located to its south-west. No clusters are detected near this nova. Also, no field star data are available for this region. 

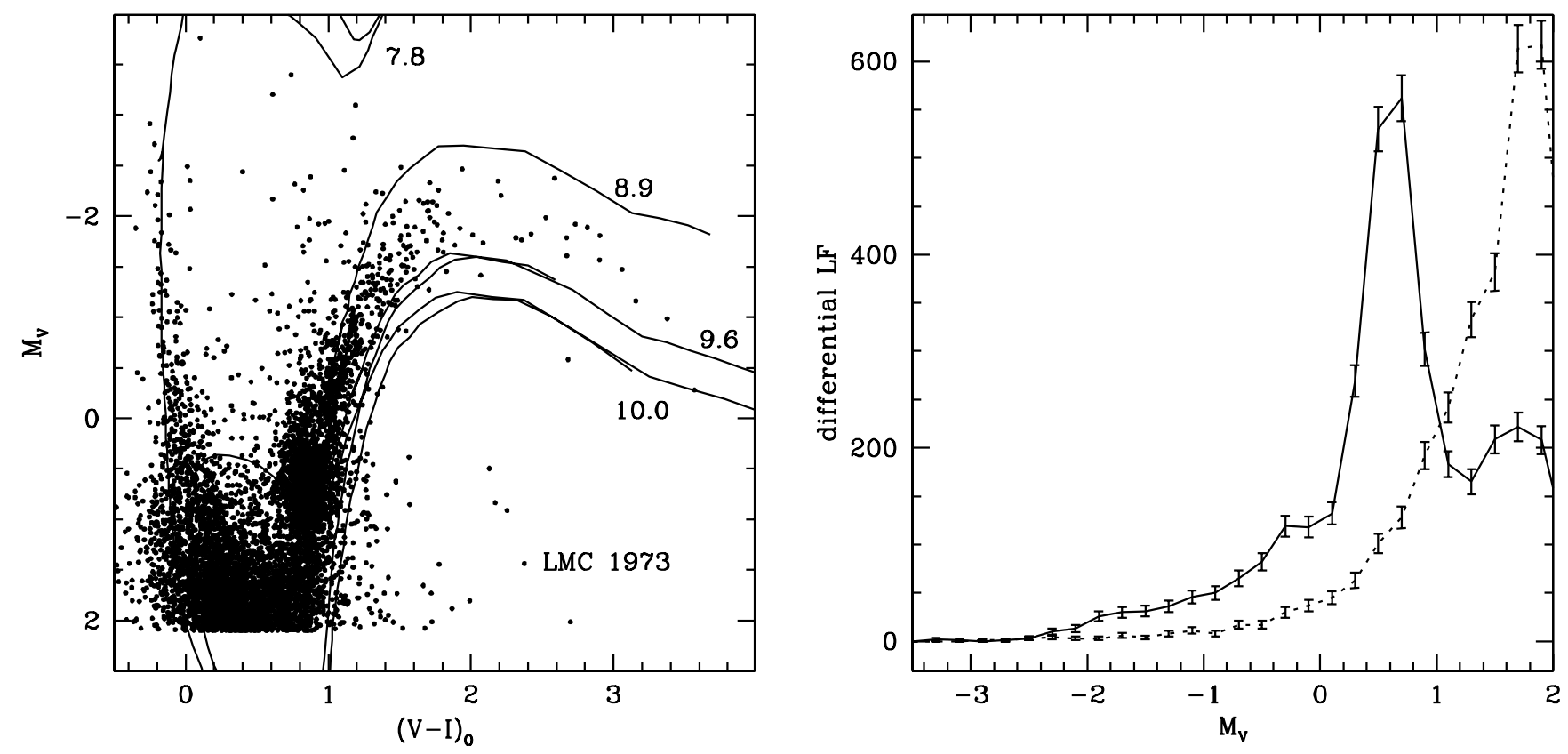

Fig. 12. Left panel: the CMD of 6739 stars within 3 arcmin from LMC 1973. The isochrones fitted to the CMD, with the corresponding value of $\log ($ age) indicated, are also plotted. Right panel: LF of the MS (dotted line) and the red giants (solid line). The error bars indicate the statistical error in the data.
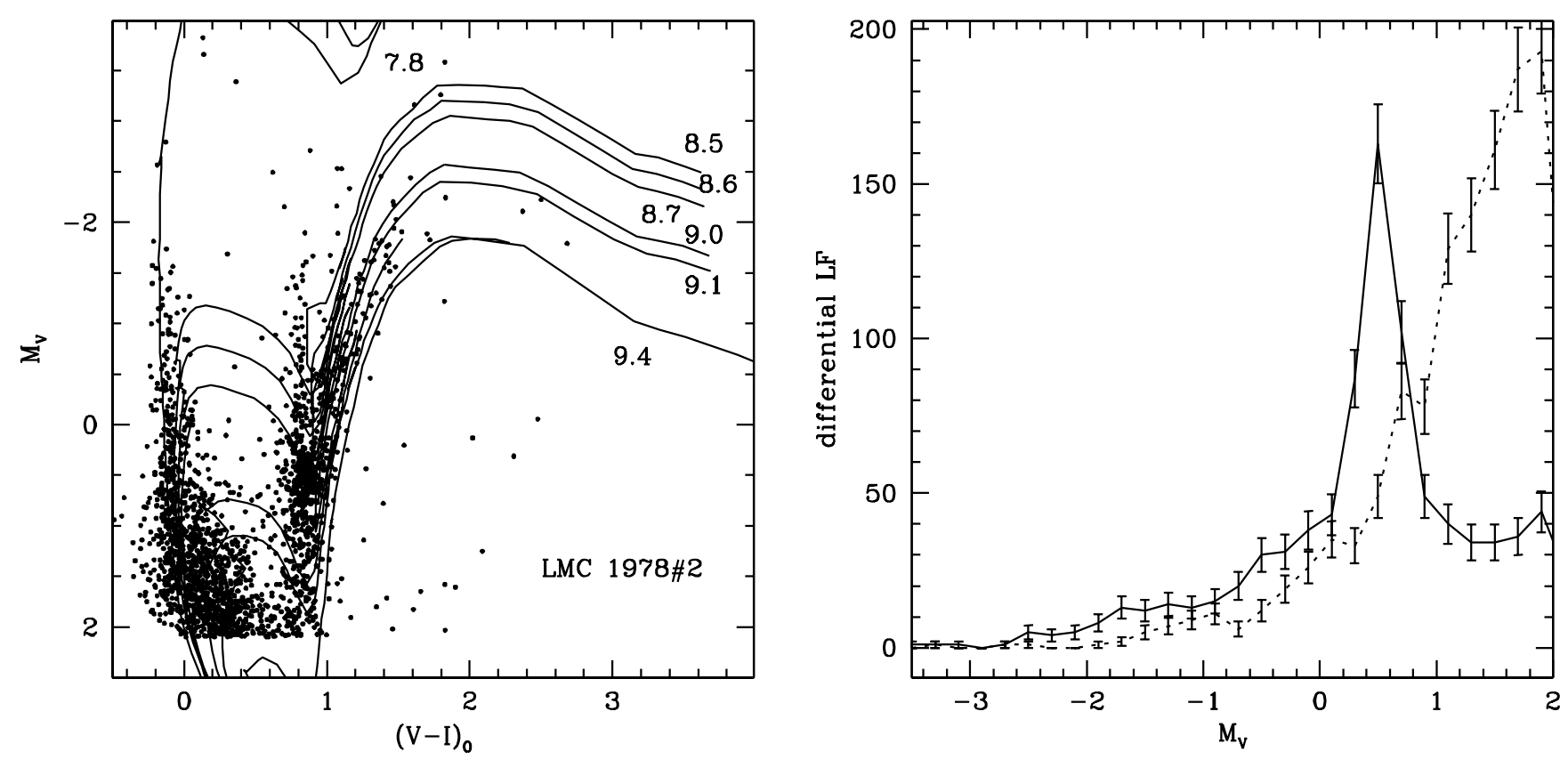

Fig. 13. Left panel: the CMD of 2160 stars from a nearby region within 5 arcmin from nova LMC 1978\#2. The isochrones fitted to the CMD, with the corresponding value of $\log ($ age) indicated, are also plotted. Right panel: LF of the MS (dotted line) and the red giants (solid line). The error bars indicate the statistical error in the data.

LMC 1978\#2 This nova is located at the north of the western edge of the Bar. There are 8 star clusters found near this nova and ages are known for 5 of them. The age ranges 100-300 Myr and $300 \mathrm{Myr}-1 \mathrm{Gyr}$ are found to have two clusters each. One cluster is found within the age range 30-100 Myr. This shows that there has been continuous formation of clusters, probably in a subdued manner.
The CMD and LF of a region close to the nova, within 5 arcmin is shown in Fig. 13. The CMD is characterised by a very a prominent RGC and a vertical extension of the RGC. Also, the MS and the RGB are well separated, except for some identifiable subgiant branches. The isochrone fits reveal that the oldest stars in this region are $2.5 \mathrm{Gyr}$ and are scantily populated. The wide MS near to $M_{V}=1.0 \mathrm{mag}$ probably belongs to 

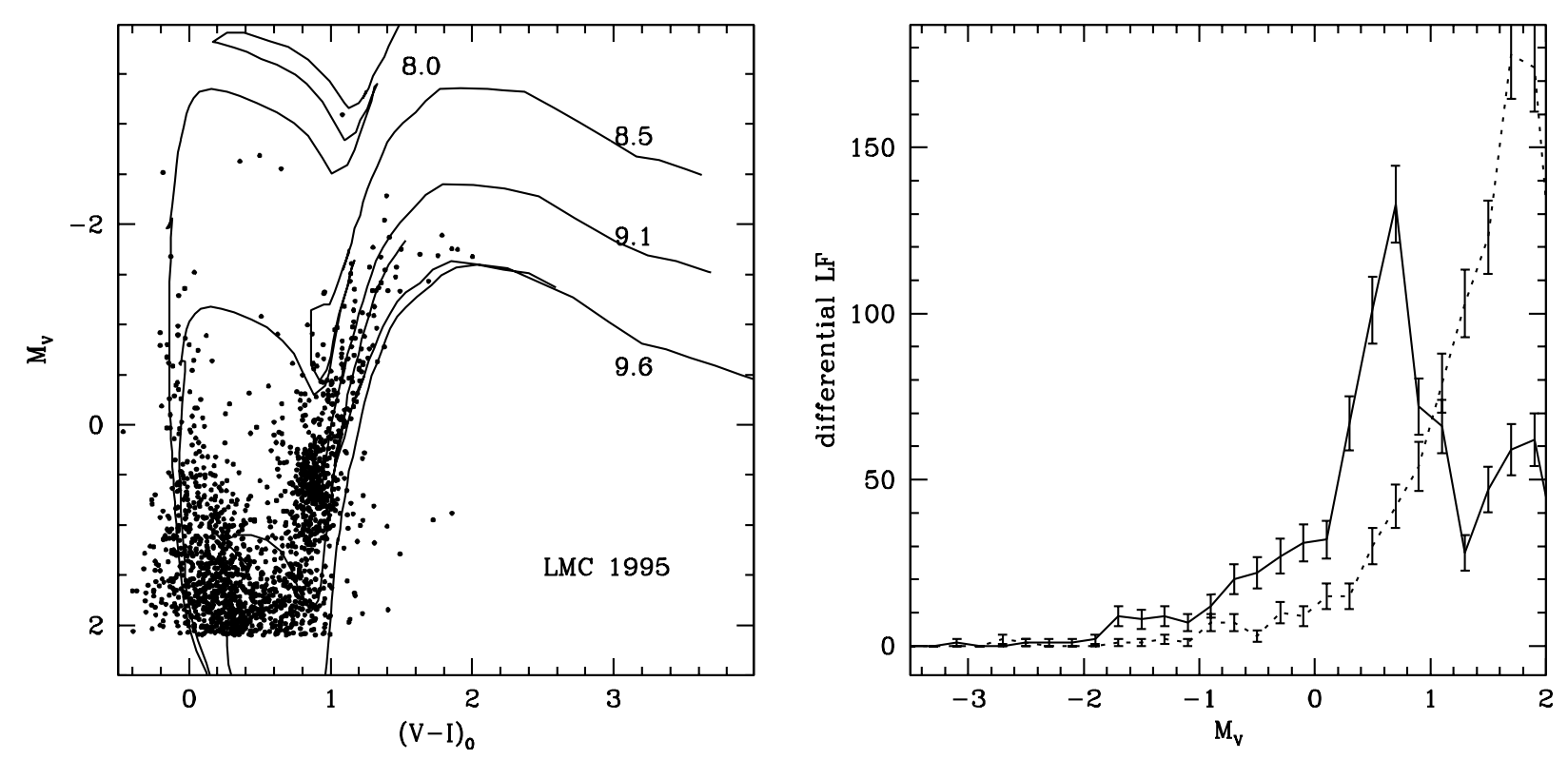

Fig. 14. Left panel: the CMD of 1797 stars within 3 arcmin from LMC 1995. The isochrones fitted to the CMD, with the corresponding value of $\log ($ age) indicated, are also plotted. Right panel: LF of the MS (dotted line) and the red giants (solid line). The error bars indicate the statistical error in the data.

1.3 Gyr old population. This isochrone also fits most of the red giants. The $1.0 \mathrm{Gyr}$ isochrone fits the RGC quite well. Isochrones with $\log (\tau)=8.5,8.6,8.7$ are seen to fit the vertical extension of the RGC. Some bright stars are seen at the top of the MS, whose ages are estimated as $63 \mathrm{Myr}$.

The RGLF shows that the RGB presents a gradually rising curve with a strong and narrowly peaked RGC. The RGB has a good number of bright stars, which are the vertical extension of the RGC.

This region has a significantly high fraction of stars $(61.8 \%)$ in the MS. The RGC has $49.0 \%$ of stars found in the RGB. The star formation which started at $2.5 \mathrm{Gyr}$, continued till 1.0 Gyr. Another star formation event has occurred at about $400 \mathrm{Myr}$, which produced four star clusters.

LMC 1995 This nova is located at the southern edge of the Bar region. This nova has been detected as a super-soft X-ray source with a 1.2 $M_{\odot} \mathrm{CO}$ white dwarf (Orio \& Greiner 1999). 27 star clusters are found in the vicinity of the nova, of which the ages are known for $22.18 \%$ of the clusters have ages within 30-100 Myr, 50\% have ages between 100-300 Myr and 32\% have ages between $300 \mathrm{Myr}-1 \mathrm{Gyr}$. This indicates that there was a burst of cluster formation some time during 100-300 Myr with a tapering cluster formation before and after this burst.

The CMD of 1797 stars within a radius of 3 arcmin of the nova is shown in Fig. 14. The isochrone fits reveal that the oldest traceable population is about 4.0 Gyr. The star formation then continued to about $1.3 \mathrm{Gyr}$. This is indicated by the fit of the isochrone to the subgiant branch location and the red giants of the RGB. A few stars can be seen to the left of the $1.3 \mathrm{Gyr}$ isochrone at the RGB, which indicates that the star formation continued until more recent times. There is a clear signature of the 300 Myr old population as indicated by the isochrone. The brightest stars in the MS are 100 Myr old.
The RGLF shows that the RGB has a bumpy profile. The RGC profile shows that there are two clumps, the brighter clump belonging to a younger population and the fainter one to a population older than that. The MSLF also shows a bumpy profile. This region has $59.0 \%$ of stars in the MS and the RGC has $43.0 \%$ of stars in the RGB.

LMC 1997 This nova is located to the north of the Bar and slightly away from it. There are two star clusters found near this nova. Both clusters have an age between 300 Myr-1 Gyr. The fact that there are only two star clusters in the vicinity indicates that the cluster formation has been very poor.

The CMD of 3514 stars from a nearby field within 8 arcmin of the nova is shown in Fig. 15. The isochrone fit to the right most part of the RGB shows that some stars belonging to about $15 \mathrm{Gyr}$ is present. The fit of the isochrone of age $\log \tau=10.2$ is shown in the CMD. The RGB is not populated with stars in the age range 15 and 3.2 Gyr. Subsequent to 3.2 Gyr, star formation seems to have been continuous for a long time, as indicated by the broad RGB, broad MS, subgiant branches and the broad and prominent RGC. The isochrones shown in the figure are for ages $15 \mathrm{Gyr}, 3.2 \mathrm{Gyr}, 1.3 \mathrm{Gyr}$ and $800 \mathrm{Myr}$. The presence of stars on to the left of the $800 \mathrm{Myr}$ isochrone at the RGB is indicative of continued star formation to more recent times. The well populated MS also supports this. The brightest part of the CMD has stars of age $100 \mathrm{Myr}$. The most luminous red giants also seem to belong to this population.

The RGLF shows that the brighter end of the RGB is very well populated. The RGC profile shows a tapering towards brighter magnitudes, and the profile itself is fairly broad and well peaked. The MSLF shows a bumpy profile.

This region has $59.7 \%$ of stars in the MS and the rest $40.3 \%$ stars in the RGB. The RGC has $52.8 \%$ of the stars in the RGB. This high value indicates that star formation was high in the 

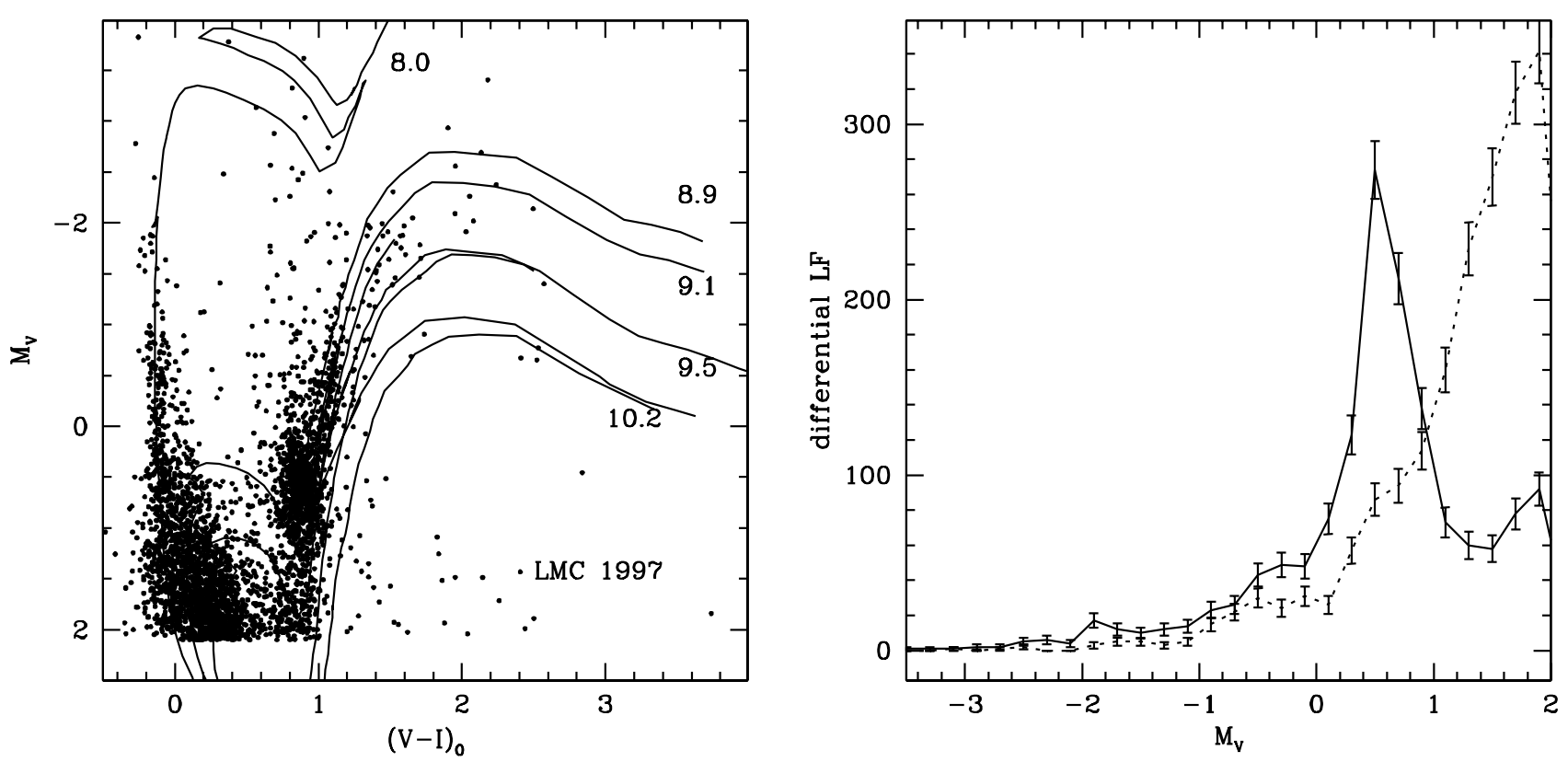

Fig. 15. Left panel: the CMD of 3514 stars from a nearby region within 8 arcmin from the nova LMC 1997. The isochrones fitted to the CMD, with the corresponding value of $\log ($ age) indicated, are also plotted. Right panel: LF of the MS (dotted line) and the red giants (solid line). The error bars indicate the statistical error in the data.
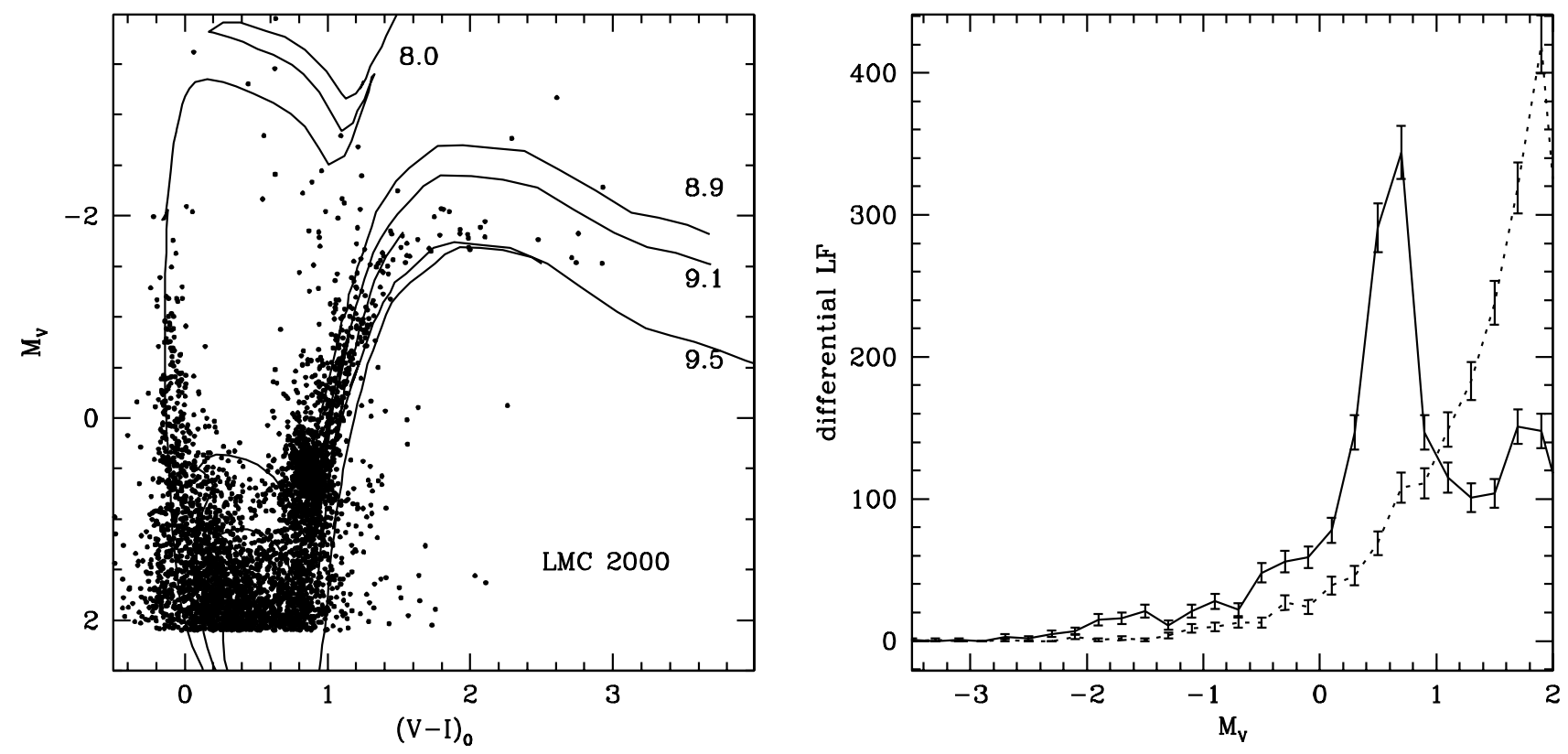

Fig. 16. Left panel: the CMD of 4054 stars within 3 arcmin from LMC 2000. The isochrones fitted to the CMD, with the corresponding value of $\log ($ age $)$ indicated, are also plotted. Right panel: LF of the MS (dotted line) and the red giants (solid line). The error bars indicate the statistical error in the data.

3.2 Gyr-800 Myr period. The star formation appears to have continued until $100 \mathrm{Myr}$, albeit a lesser vigour. This region has some trace of the very old population of the LMC.

LMC 2000 This nova is located a to the south of the Bar. There are 5 star clusters found near this nova. All the five have age estimates, with one cluster each in the age ranges $300 \mathrm{Myr}-$ 1 Gyr; 1 Gyr and beyond respectively. Three clusters have ages within 100-300 Myr.
The CMD of 4054 stars within a radius of 3 arcmin from the nova is show in Fig. 16. The CMD shows a well populated RGB, MS and a prominent RGC. The CMD does not show any trace of an old population. The star formation probably started at about 3.2 Gyr, as seen by the isochrone fit to the CMD. It continued till $800 \mathrm{Myr}$, as seen by the isochrones of ages 1.3 Gyr and 800 Myr. The star formation then stopped or continued at very low rates till $100 \mathrm{Myr}$ ago. The MS between the $800 \mathrm{Myr}$ and $100 \mathrm{Myr}$ turn-offs is only scantily populated indicating a low star formation rate. 
Table 4. LMC novae: Age estimates of the neighbourhood stellar population.

\begin{tabular}{|c|c|c|c|c|}
\hline Nova & $\begin{array}{c}\text { old stellar } \\
\text { population } \\
(\mathrm{Gyr})\end{array}$ & $\begin{array}{c}\text { intermediate } \\
\text { population } \\
(\mathrm{Gyr})\end{array}$ & $\begin{array}{c}\text { moderately } \\
\text { young } \\
(\mathrm{Myr})\end{array}$ & $\begin{array}{l}\text { young } \\
\text { (Myr) }\end{array}$ \\
\hline \multicolumn{5}{|l|}{ ONeMg novae } \\
\hline LMC 1981 & 10 & $3.2-1.3$ & - & 125 \\
\hline LMC 1988\#2 & 10 & $3.2-1.6$ & 400 & 79 \\
\hline LMC 1990\#1 & 10 & \multicolumn{2}{|c|}{$4.0 \mathrm{Gyr}-250 \mathrm{Myr}$} & 40 \\
\hline \multicolumn{5}{|l|}{ Fast } \\
\hline LMC 1977\#2 & 10 & $4.0-1.0$ & 300 & 40 \\
\hline LMC 1987 & 10 & $3.2-1.3$ & 300 & 63 \\
\hline LMC 1992 & - & $2.5-1.3$ & 500 & 63 \\
\hline \multicolumn{5}{|c|}{ Moderately fast } \\
\hline LMC 1936 & - & $2.0-1.0$ & 300 & 63 \\
\hline LMC 1988\#1 & - & $2.5-1.0$ & 300 & 100 \\
\hline \multicolumn{5}{|l|}{ Slow } \\
\hline LMC 1948 & & $10 \mathrm{Gyr}-630 \mathrm{Myr}$ & & 100 \\
\hline \multicolumn{5}{|c|}{ Speed class unknown } \\
\hline LMC 1970\#1 & - & $3.2-1.0$ & 300 & 100 \\
\hline LMC 1973 & 10 & $4.0-0.80$ & - & 63 \\
\hline LMC 1978\#2 & - & $2.5-1.0$ & 400 & 63 \\
\hline LMC 1995 & - & $4.0-1.3$ & 300 & 100 \\
\hline LMC 1997 & 15 & $3.2-0.80$ & - & 100 \\
\hline LMC 2000 & - & $3.2-0.80$ & - & 100 \\
\hline
\end{tabular}

The RGLF shows a slowly rising profile, with a relatively sharp RGC profile. MSLF is seen to be a smooth profile. The MS contains $55.9 \%$ of total number of stars in the region and the rest are in the RGB. The RGC contains $42.9 \%$ of stars in the RGB.

\section{Discussion}

\subsection{Distribution and population of novae in the LMC}

A study of the distribution of novae by van den Bergh (1988) showed that the nova population followed the old disk population. Further, van den Bergh did not find any nova in the Bar region and concluded the Bar in the LMC to be a recent phenomena. Subsequent to van den Bergh's study, 10 novae have been detected. The projected locations show that, among these ten, four are in the Bar and four are very close to the Bar. This shows that the population responsible for producing novae may also be present in the Bar region.

The LMC seems to have a high population of fast novae, $72.2 \%$ of the novae with known speed classes are fast novae. The slow novae are only $11.1 \%$. Duerbeck (1990), Della Valle et al. (1992) and Yungelson et al. (1997) have shown the existence of two nova populations in the Galaxy and M31: fast, bright disk novae and slow, faint bulge novae. The disk novae probably belong to a relatively younger parent population having higher white dwarf masses. The larger population of fast novae implies a predominantly disk population in the LMC. This is consistent with the fact that in the LMC the old halo population is a minority population, whereas the intermediate age population of a few Gyr is the dominant population.

The locations covered by the fast and the moderately fast novae are indicative of the extension of the LMC disk. It is of interest to note that both the identified slow novae are located within the regions of fast novae. The recurrent nova is also seen to be located not very far from the location of other novae.

\subsection{Star formation history}

The star formation history of nearby regions around 15 novae are studied here. The results derived in the previous section are presented in Table 4.

Traces of stellar population belonging to the old population of the LMC is found in 8 regions. In the remaining 7 regions, no old population is found. This indicates a low density of the old population in these regions.

It can be seen from Table 4 that the intermediate age population seems to be in the age range 4.0-1.0 Gyr, with minor variations depending on the region. No detectable population is seen between 4.0-10.0 Gyr, except in one region, around LMC 1948. 

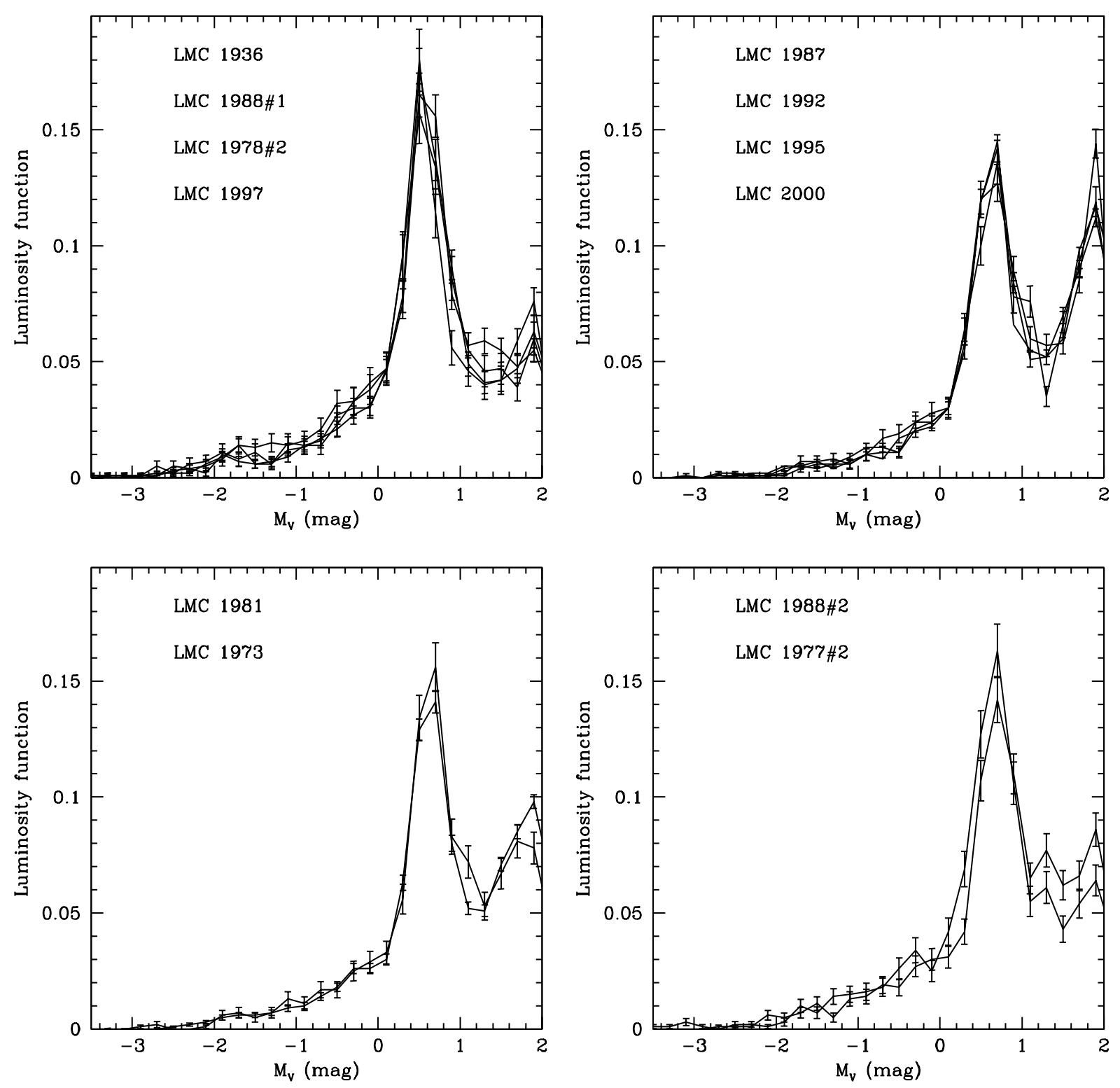

Fig. 17. The regions having similar profile of RGLF are grouped and plotted. The error bars indicate the statistical error in the data.

Among the intermediate stellar population, the most prominent feature is the RGC. From the data presented in Table 3, it is seen that most of the regions have around $40 \%-50 \%$ of the total stars in the RGB, with more than $40 \%$ of them in the RGC. It is evident from the table that a higher fraction of RGB stars does not imply a higher fraction of RGC stars. In can also be noticed that the fraction of RGC stars seem to be similar in the case of a some regions, indicative of some groups. This could indicate similar star formation events, if the RGLF has similar profiles. The RGLFs of the regions having similar values for the RGC star fraction were over plotted and some were found to have very similar profiles. These are shown in Fig. 17. The RGLFs are normalised to the total number of RGB stars for comparison.

The top left panel in the figure shows the regions around the novae, LMC 1936, LMC 1988\#1, LMC $1978 \# 2$ and LMC 1997. The RGLF in these regions is characterised by a very prominent RGC clump with a narrow and peaked profile for the clump. The first two regions in this group are located near moderately fast novae and the other two are around novae without speed class estimates. The first three regions are located in the north-western part of the LMC, whereas the fourth one is located to the south-east of the Bar. Also, the first three regions experienced major star formation event slightly later, at 2.5 Gyr, which ended by about 1 Gyr. A few studies on the stellar population in the northern region of the LMC also show similar results. For e.g. the SFH of two northern regions studied by Geha et al. (1998) using HST data, showed an enhancement in star formation at about 2 Gyr ago. Similarly, the SFH of two other northern LMC fields studied by Dolphin (2000) provide evidence for an increment in star formation about 2.5 Gyr ago.

The top right panel in Fig. 17 shows that the RGLF of stars in the regions around the novae, LMC 1987, LMC 1992, LMC 1995 and LMC 2000. These profiles are characterised by a comparatively broader RGC clump profile, with a shorter peak. Among these four regions, the first two are located 


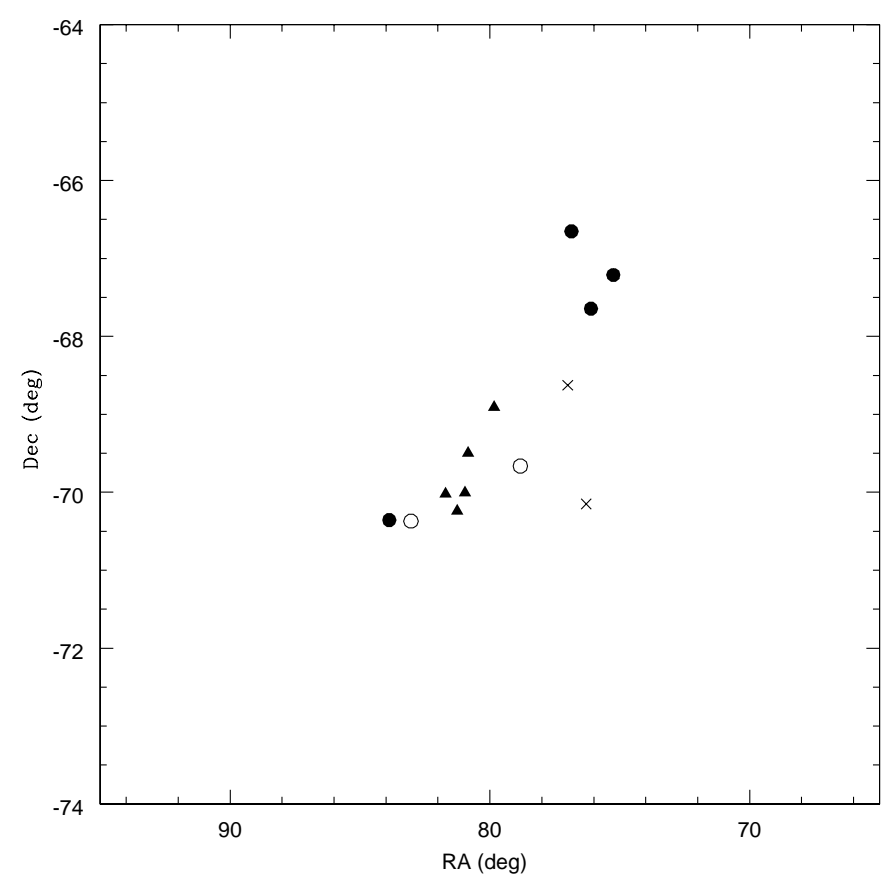

Fig. 18. The location of regions considered in Fig. 17 are plotted here. Each group of regions with similar RGLF profiles is identified by the same symbol.

around fast novae, whereas the speed class is not known for the novae in the other two regions. It is of interest to note that all these four regions are located close to the center of the Bar, at least in the projected view. Especially, the regions around novae LMC 1987, LMC 1995 and LMC 2000 are located very close to one another.

The two plots in the upper portion of the Fig. 17 seem to indicate that there may be a difference in the RGLF of the regions surrounding fast novae and moderately fast novae. This is only an indication as the known number of fast and moderately fast novae is quite small.

The bottom left panel in Fig. 17 shows that the RGLF of regions around LMC 1981 and LMC 1973 are similar. These are characterised by a slightly flatter peak. The first region is around a fast nova, while the speed class of the nova in the second region is not estimated. The bottom right panel shows the RGLF of regions around LMC 1988\#2 and 1977\#2. The RGLF is characterised by a relatively broad and sharp RGC clump. These two regions are located around fast novae.

In order to substantiate the results of the above discussion, the locations of the groups showing similar RGLF profiles are shown in Fig. 18. It can be seen from the figure that the novae regions near the central region of the Bar have similar RGLF profiles, indicating that these regions might have had a similar SFH. A look at the MSLF of these five regions indicate that the MSLF profiles are also very similar supporting the fact that these regions might have had a similar SFH.

A striking feature noticed in the analyses is that the stellar population near LMC 1948 is different from those seen in other regions. The region around LMC 1948 seems to indicate the presence of old stellar population, much older than 4.0 Gyr, unlike what is seen in other regions. A careful look at the CMDs presented by Udalski et al. (2000) of the LMC fields indicate the presence of regions which have a SFH very similar to that around nova LMC 1948. The HST data of the field region near the star cluster NGC 2019, which is located within the Bar, presented by Olsen (1999), also shows a very broad RGC and scattered RGB similar to the region around nova LMC 1948. This region was also estimated to have had an enhanced star formation 5-8 Gyr ago. It is thus quite likely that pockets of regions with a SFH similar to that around LMC 1948 can be found within or near the Bar.

The moderately young population of a few hundred Myr is seen in 10 out of 15 regions studied here. This is in the age range 250-500 Myr, with 6 out of the 10 regions having $300 \mathrm{Myr}$ age population. The youngest stellar population is in the range 50-100 Myr, except in the case of three regions. None of the regions show stars younger than $40 \mathrm{Myr}$.

The metallicity of the isochrones used is $Z=0.008$, which is equivalent to $[\mathrm{Fe} / \mathrm{H}]=-0.40$. This value of metalicity is found to fit all the 15 regions. Hence we find that the metalicity of the regions studied is more or less the same for the intermediate age stars and for stars younger than that.

Red Clump Stars The red clump stars correspond to the corehelium burning stars. When there is a heterogeneous population of stars, the luminosity of the red clump stars is dependent on the age and metallicity of the underlying stellar population (Girardi \& Salaris 2001). Hence the age of the RGC stars can be estimated from their luminosity, once the metallicity is known. Therefore the peak luminosity in the RGC profile can be used to estimate the approximate age of the RGC stars. As the $Z=0.008$ is found to fit the CMDs, this value of $Z$ can be assumed for the red clump stars also. The relation between the age and the luminosity of the red clump stars for $Z=0.008$ given in Girardi \& Salaris (2001) is used to estimate the approximate age of the red clump stars in the regions studied here. The value of $Z$ assumed here is consistent with the $[\mathrm{Fe} / \mathrm{H}]$ values used by Girardi \& Salaris (2001) for the LMC regions. It can be seen that the peak luminosity of the RGC profile is in the range $M_{V}=0.5-0.7 \mathrm{mag}$. The RGLF indicates that either the RGC peaks at both these magnitudes or at one of these. From Table 1 in Girardi \& Salaris (2001), it can be seen that this range in luminosity corresponds to an age range 3-1 Gyr approximately. Girardi \& Salaris (2001) mention that the bulk of the red giants in the LMC are $\leq 3$ Gyr. Therefore the red clump age range estimated here is within the expected range.

Star clusters Star clusters within a radius of 30 arcmin from the nova location are studied here with the assumption that star cluster formation episodes could be linked to star formation episodes. Most of the clusters for which age estimates are available appear to have formed between $100 \mathrm{Myr}$ and $300 \mathrm{Myr}$ or between $300 \mathrm{Myr}$ and $1 \mathrm{Gyr}$. Only in one case, in the vicinity of LMC 1990\#1, a significant number of star clusters appear to have formed before $1 \mathrm{Gyr}$.

The star cluster data presented in Table 2 shows that though most of the regions have star clusters, the ages are known for a significant fraction in only some regions. Considering only 
regions for which ages are known for good fraction of clusters, we find that 7 out of 13 regions $(53.9 \%)$ have clusters aged more than 1 Gyr. As the LMC is seen to be devoid of star clusters within the age range $3 \mathrm{Gyr}$ to $10 \mathrm{Gyr}$, and only a few very old clusters are known, it is assumed that these clusters fall in the $1 \mathrm{Gyr}-2.5 \mathrm{Gyr}$ age range. The intermediate age star cluster formation episodes are correlated with star formation episodes in only $\sim 54 \%$ of the regions. It thus appears that star clusters are not good tracers of the intermediate age stellar population in the LMC.

The star cluster formation seems to be very well correlated to the star formation events in the 100-300 Myr range. A good number of star clusters are found in this age range, wherever there has been a noticeable stellar population of the same age range. Therefore, the star clusters are seen to be good tracers of star formation in the age range 100-300 Myr.

The youngest cluster population seen is younger than $30 \mathrm{Myr}$, and is found in the vicinity of 7 novae. On the other hand, no stellar population younger than $40 \mathrm{Myr}$ is seen in any of the novae regions. Therefore, we do not see any correlation between cluster formation and star formation in this age range. Hence the star clusters are not a good tracers of stellar population younger than $30 \mathrm{Myr}$.

\subsection{Probable parent population of novae}

The parent population of the novae is believed to belong to the intermediate age population. In the nova regions studied here, we find that the star formation began a few Gyr ago, though there are traces of old stellar population. The population which is the outcome of this star formation event is likely to be the parent population of the novae. The star clusters do not appear to be good tracers of the stellar population in the intermediate age range, as seen in the previous section. Hence only the intermediate age stellar population and their ages are considered to identify the probable parent population of the LMC novae. The stellar population considered is the projected population near the novae. As the LMC is known to have a thin disc, we assume that the depth of the LMC in the line of sight to be small. Therefore the assumption that the nova and the projected stellar population are not very much spatially separated is valid. In this study, we have analysed the stellar population around 15 regions on the face of the LMC and identify the intermediate population which is common to the regions belonging to each type of nova. This approach thus more or less eliminates any biases arising due to the projection effect.

Of the six fast nova regions studied here, we find that in three regions, the intermediate age population has an upper age limit of 3.2 Gyr, two regions have 4.0 Gyr and one has 2.5 Gyr. The lower limit is $1.3 \mathrm{Gyr}$ for three, $1.6 \mathrm{Gyr}$ for one and $1 \mathrm{Gyr}$ for one. As one region (LMC 1990\#1) has continued star formation, the lower age limit is not possible to identify. This implies that the parent population of fast novae lie in the age range 4.0-1 Gyr. Assuming the parent population to be in the age range which is in common in all the six regions, the age range of the parent population of the fast novae is likely to be 3.2-1.6 Gyr.
The regions around the two moderately fast novae studied here indicate a parent population in the age range 2.5-1.0 Gyr.

The region around only one slow novae has been studied. The striking differences between this region and those around the fast and moderately fast novae are: (a) continuous star formation between $10 \mathrm{Gyr}-630 \mathrm{Myr}$, and (b) presence of stars belonging to the age range $4-10$ Gyr. As there is only one slow nova in the LMC for which field star data are available, we looked into the projected stellar population around a slow nova in Small Magellanic Cloud (SMC); SMC 1994. SMC 1994 was detected in the EROS microlensing survey, located in the core of SMC (de Laverny et al. 1998). Detailed observations of this nova indicated it to be a slow nova, with properties similar to the slow, dust forming Galactic novae. The region in which SMC 1994 occurred has been scanned by the OGLE project in their survey of the SMC (Udalsky et al. 1998). An analyses of the CMD of the field stars in the nova neighbourhood shows that the region had a SFH similar to the LMC 1948 region and that the RGB stars span a range of 1-10 Gyr in age. The above facts imply that the progenitors of slow novae probably belong to an older population, consistent with the idea of slower, fainter, bulge novae in the Galaxy and M31.

The age estimates for the novae for which the speed class is not known, show that the common age range is 1.3-2.5 Gyr. If one includes all the ranges, then the age of the intermediate age population is in the range $4 \mathrm{Gyr}-800 \mathrm{Myr}$.

Combining the results obtained for 14 regions around novae, (excluding the region around slow nova), we see from Table 4 , that $28.6 \%$ (4 out of 14 regions) of the regions have $4 \mathrm{Gyr}, 42.9 \%$ (6 out of 14) have $3.2 \mathrm{Gyr}, 21.4 \%$ (3 out of 14) have $2.5 \mathrm{Gyr}$ and $7 \%$ have $2.0 \mathrm{Gyr}$ as the upper limit for age of the intermediate age population. The lower limit for the age of the intermediate age population is found to be $1.6 \mathrm{Gyr}$ for $7 \%$ of the regions, $1.3 \mathrm{Gyr}$ for $28.6 \%$ of the regions, $1.0 \mathrm{Gyr}$ for $42.9 \%$ of the regions and $800 \mathrm{Myr}$ for $21.4 \%$ of the regions. The important limit derived in this study is the upper age limit of the parent population of novae in the LMC, which is 4 Gyr. The lower age limit for the parent population of the novae is more likely to be 1 Gyr. If we consider the most likely limit, then the progenitor age range is likely to be between 3.2-1.0 Gyr. The progenitor for the slow novae is likely to originate from the population, in the age range $1-10 \mathrm{Gyr}$.

In the previous section, it was found that the red clump stars in the LMC are likely to be in the age range 3-1 Gyr and this is very similar to the age range for the progenitors of the fast and moderately fast novae. It is very likely that the progenitors of fast and moderately fast novae and red clump stars are similar in age and metallicity.

Applying standard models for the formation and evolution of cataclysmic variables, Kolb \& Stehle (1996) determined the age structure for a model population of Galactic cataclysmic variables. Their model predicts that there are two age groups, above and below the orbital period gap. The systems above the period gap are younger than 1.5 Gyr and those below the gap are of age 3-4 Gyr. The possible age for the nova population derived in this study is in good agreement with the age estimates of Kolb \& Stehle (1996), keeping in mind that their estimates are for the Galactic cataclysmic variables. 
This indicates that the progenitor age range as found in the LMC is very similar to that estimated for our Galaxy. As the novae in the LMC are predominantly disk novae, the progenitors of disk novae are likely to be in the age range 3.2-1 Gyr.

Other galaxies The SFH around the nova regions in the LMC may be compared with the SFH of other galaxies where novae have been detected, in particular, M 33 which is also a disk dominated galaxy. Based on a study of the star clusters in M 33, Chandar et al. (1999) and Ma et al. (2001) find that M 33 experienced an enhancement in cluster formation around a few Gyr and that most of these clusters are metal poor. If we assume that the intermediate age cluster formation is correlated with that of the stellar population, then it appears that the parent population of novae in M33 are similar to those in the LMC. It is of further interest to note that NGC 5128, an elliptical galaxy where a fair number of novae have been detected (Ciardullo et al. 1990), shows a significant presence of intermediate age AGB stars as indicated by a recent study based on VLT observations (Rejkuba et al. 2001).

\section{Summary and conclusions}

The underlying population in the location of novae in the LMC are studied in an attempt to understand the common characteristics of the nova regions and thereby tried to identify the parent population of novae. There has been no previous attempt to directly study the stellar population in the neighbourhood of novae.

The LMC is an ideal galaxy to undertake such a study as a good number of novae are identified in the LMC and the field population has been very well studied through many recent surveys. The stellar population and the star clusters near the novae are used for this study. The local population of the novae are studied using the OGLE II field star data and the star clusters are obtained from catalogues. The star formation history in the location of 15 novae are studied using CMDs, LFs and comparing the fraction of stars in the MS, RGB and RGC. The episodes of cluster formation are determined by grouping the clusters in age. The above analysis leads to the following conclusion:

1. The LMC has $72.2 \%$ novae in the fast category and $11.1 \%$ in the slow category. This suggests that the fraction of the intermediate age stellar population to which the fast novae belong is correspondingly substantial, when compared to the fraction of the old stellar population which are responsible for the slow novae.

2. The fast novae (including the ONeMg type) and the moderately fast novae have a very similar parent population. Also, the SFH of intermediate age stars near these novae are very similar, while that around the slow nova is very different.

3. The upper age limit of the stellar population to which the fast and moderately fast novae belong is likely to be 3.2 Gyr, while it cannot be higher than 4 Gyr. The lower age limit of the parent nova population is most likely to be 1.0 Gyr. This is in good agreement with the age estimates of Kolb \& Stehle (1996) for Galactic cataclysmic variables.
4. The region around slow nova shows continuous star formation between 1-10 Gyr, with a good fraction belonging to the 4-10 Gyr population, consistent with the idea that the progenitors of slow novae belong to an older population.

Acknowledgements. We thank R. Sagar, H.W. Duerbeck and the referee for useful comments and suggestions.

\section{References}

Andrillat, Y., \& Dennefeld, M. 1983 A\&A, 124, 143

Ardeberg, A., Gustafsson, B., Linde, P., \& Nissen, P.-E. 1997, A\&A, 322,13

Bertelli,G., Bressan, A., Chiosi, C., Fagotto, F., \& Nasi, E. 1994, A\&AS, 106, 275

Bica, E., Claria, J. J., Dottori, H., Santos, J. F. C. JR., \& Piatti, A. E. 1996, ApJ, 102, 57

Bica, E., Schmitt, H. R., Dutra, C. M., \& Oliveira, H. L. 1999, AJ, 117,238

Buscombe, W., \& de Vaucouleurs, G. 1955, Observatory, 75, 170

Butcher, H. 1977, ApJ, 216, 372

Capaccioli, M., Della Valle, M., D’Onofrio, M., \& Rosino, L. 1989, AJ, 97, 1622

Capaccioli, M., Della Valle, M., D’Onofrio, M., \& Rosino, L. 1990, ApJ, 360, 63

Canterna, R., \& Thompson, L. F. 1981, PASP, 93, 581

Chandar, R., Bianchi, L., \& Ford, H. C. 1999, ApJ, 517, 668

Ciardullo, R., Ford, H. C., Williams, R. E., Tamblyn, P., \& Jacoby, G. H. 1990, AJ, 99, 1079

Ciardullo, R., Ford, H. C., Neill, J. D., Jacoby, G. H., \& Shafter, A. W. 1987, ApJ, 318, 520

de Laverny, Beaulieu, J. P., Asplund, M., et al. (EROS coll.) 1998, A\&A, 335, L93

Della Valle, M. 1991, A\&A, 252, 9

Della Valle, M., Bianchini, A., Livio, M., \& Orio, M. 1992, A\&A, 266, 232

Della Valle, M., \& Livio, M. 1995, ApJ, 452, 704

Della Valle, M., \& Duerbeck, H. W. 1993, A\&A, 271, 175

Della Valle, M., Rosino, L., Bianchini, A., \& Livio, M. 1994, A\&A, 287, 403

Dolphin, A. E. 2000, MNRAS, 313, 281

Duerbeck, H. W. 1984, Ap\&SS, 99, 363

Duerbeck, H. W. 1990, in Physics of Classical Novae, ed. A. Casatella, \& R. Viotti (Berlin: Springer), 34

Geha, C. M., et al. 1998, ApJ, 115, 1045

Gilmore, A. C. 2002, IAUC, 7853

Girardi, L., \& Salaris, M. 2001, MNRAS, 323, 109

Hachisu, I., Kato, M., Kato, T., \& Mastsumoto, K. 2000, ApJ, 528, L97

Hatano, K., Branch, D., \& Fisher, A. 1997, ApJ, 487, L45

Hatano, K., Branch, D., Fisher, A., \& Starrfield, S. 1997, MNRAS, 290, 113

Kato, M. 1997, in Cosmic Chemical Evolution, IAU Symp., 187, 20

Kilmartin, P., \& Gilmore, A. C. 2002, IAUC, 7847

Kolb, U., \& Stehle, R. 1996, MNRAS, 282

Liller, W. 2002, IAUC, 7841

Ma, J., Zhou, X., Kong, X., et al. 2001, AJ, 122, 1796

Olsen, K. A. 1999, AJ, 117, 2244

Orio, M., \& Greiner, J. 1999, A\&A, 344, 130

Pietrzynski, G., Udalski, A., Kubiak, M., et al. 1999, Acta Astron., 49, 521

Pietrzynski, G., \& Udalski, A. 2000, Acta Astron., 50, 337

Pritchet, C. J., \& van den Bergh, S. 1987, ApJ, 318, 507 
Rejkuba, M., Minniti, D., Silva, D. R., \& Bedding, T. R. 2001, A\&A, Starrfield, S., Truran, J. W., \& Sparks, W. M. 2000, New Astron. Rev., 379,781 44,81

Schwarz, G. J., Hauschildt, P. H., Starrfield, S., et al. 1998, MNRAS, Udalski, A., Szymanski, M., Kubiak, M., et al. 2000, Acta Astron., 50, 300,931 307 (OGLE II data)

Schwarz, G. J., Shore, S. N., Starrfield, S., et al. 2001, MNRAS, 320, 103

Udalski, A., Soszynski, I., Szymanski, M., et al. 1999, Acta Astron., 49, 223

Searle, L., Wilkinson, A., \& Bagnoulo, W. 1980, ApJ, 239, 803

Sekiguchi, K., Kilkenny, D., Winkler, H., \& Doyle, J. G. 1989, MNRAS, 241, 827

Udalski, A., Szymanski, M., Kubiak, M., et al. 1998, Acta Astron., 48, 147

van den Bergh, S. 1988, PASP, 100, 1486

Sekiguchi, K., Caldwell, J. A. R., Stobie, R. S., \& Buckley, D. A. H. 1990, MNRAS, 245, 28

Vanlandingham, K. M., Starrfield, S., Shore, S. N., \& Sonneborn, G. 1999, MNRAS, 308, 577

Shore, S. N., Sonneborn, G., Starrfield, S. G., et al. 1991, ApJ, 370, 193

Williams, R. M., Chu, Y-H., Dickel, J. R., et al. 1999, ApJS, 123, 467

Yungelson, L., Livio, M., \& Tutukov, A. 1997, ApJ, 481, 127

Starrfield, S., Sparks, W. M., \& Truran, J. 1985, ApJ, 291, 136 